
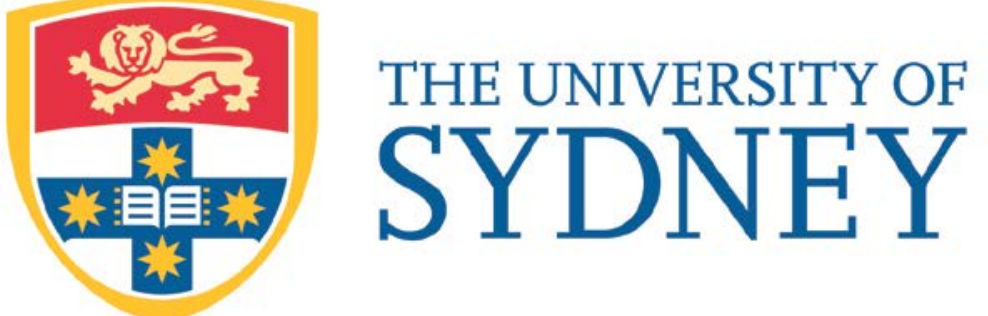

Economics Working Paper Series

$2016-14$

A Flexible Generalised Hyperbolic option Pricing

Model and its Special Cases

Claudia Yeap, Simon S. Kwok, and S. T. Boris Choy 


\title{
A Flexible Generalised Hyperbolic Option Pricing Model and its Special Cases
}

\author{
Claudia Yeap ${ }^{a}$, Simon S. Kwok ${ }^{b}$, and S. T. Boris Choy ${ }^{a}$ \\ ${ }^{a}$ Discipline of Business Analytics, The University of Sydney Business School, Australia \\ ${ }^{b}$ School of Economics, The University of Sydney, Australia
}

Address correspondence to Simon S. Kwok,

School of Economics,

The University of Sydney,

NSW 2006 Australia,

phone: +61293516607, or

email: simon.kwok@sydney.edu.au.

This version: September 11, 2017

Acknowledgement: We deeply thank Fabio Trojani, the Co-editor, and two anonymous referees for their constructive suggestions which led to substantial improvement on the paper. We also thank those who gave valuable comments to our work at the EcoSta2017 Conference and at a seminar at the University of Sydney Business School. All remaining errors are ours.

This article has been accepted for publication in the Journal of Financial Econometrics, published by Oxford University Press: https://academic.oup.com/jfec/article-abstract/doi/10.1093/jjfinec/nbx030/4555529/A-FlexibleGeneralized-Hyperbolic-Option-Pricing 


\title{
A Flexible Generalised Hyperbolic Option Pricing Model and its Special Cases
}

\author{
Claudia Yeap ${ }^{a}$, Simon S. Kwok ${ }^{b, \star}$, and S. T. Boris Choy ${ }^{a}$ \\ ${ }^{a}$ Discipline of Business Analytics, The University of Sydney Business School, Australia \\ ${ }^{b}$ School of Economics, The University of Sydney, Australia \\ * Address correspondence to simon.kwok@sydney.edu.au.
}

June 2, 2017

\begin{abstract}
We study a generalised hyperbolic (GH) time-changed Lévy process for option pricing and examine six three-parameter special cases: the variance gamma (VG) model of Madan, Carr and Chang (1998), t, hyperbolic, normal inverse Gaussian, reciprocal hyperbolic, and normal reciprocal inverse Gaussian option pricing models. We study the GH model's moment properties of the associated risk-neutral distribution of logarithmic spot returns, and obtain an explicit pricing formula for European options facilitated by the time-change Lévy process construction. Using S\&P 500 Index European options during low and high volatility sample periods, we compare the GH model empirically with existing benchmark models such as the finite-moment log-stable (FMLS) model and the Black-Scholes model. The GH model offers the best in- and out-of-sample performance overall, and the $t$ model special case generally performs better than the better known VG model. We also present a stochastic volatility extension of the GH model.
\end{abstract}

Key words: generalised hyperbolic, $t$ distribution, variance gamma, time deformation, Lévy processes

JEL classification: C58, G13

Empirical option prices indicate that the likelihood of extreme logarithmic stock returns is higher than that implied by the Black-Scholes model. Option prices also reveal that market participants pay more to protect themselves from losses than to pursue gains of equivalent magnitude. The implication is that the risk-neutral distribution of log-returns exhibits excess kurtosis and negative skewness (??). These two digressions from the normality assumption (??) are in part responsible for the poor empirical pricing performance of the Black-Scholes model. To combat this deficiency, the generalised hyperbolic $(\mathrm{GH})$ distribution may be used to improve option pricing as it accommodates skewness and thicker, semi-heavy tails (?).

In this paper, we focus on addressing the challenges faced by GH option pricing in the setting in which spot returns are independent. Our contribution to this field is to propose a new form of the GH option pricing model, the flexible GH model, which contains four free parameters and can be conveniently estimated. In addition, we present six three-parameter option pricing models as special cases of the flexible GH model, including the variance gamma (VG), $t$, hyperbolic, normal inverse Gaussian (NIG), reciprocal hyperbolic, and normal reciprocal inverse Gaussian models. With the exception of the VG model proposed by ?, the remaining five option pricing models are innovations of this paper.

To construct the flexible GH option pricing model, we generalise the Black-Scholes model by the time change (or subordination) method (?). More specifically, the risk-neutral dynamics of the log-returns is modelled by a drifted Brownian motion subordinated by a stochastic time deformation process, which is defined to be a generalised inverse Gaussian (GIG) process (?). This construction generates a class of pure-jump, infinite activity 
processes (?) 1 A typical sample path of the resulting GH process jumps infinitely many times within an infinitesimally small interval, thus enabling the model to capture both discrete and continuous asset price movements (?).

The GH process constructed this way belongs to the time-changed Lévy process family (?, ?, ?). Taking this time change approach is especially effective for developing more plausible and tractable models for asset pricing ${ }^{2}$ In terms of derivative pricing, the methodology lends itself to the derivation of an explicit pricing formula for European options, as the characteristic function of the logarithmic spot price can be conveniently obtained. Furthermore, with a judicious choice of the Lévy process and the time deformation process, the modeller can easily incorporate stochastic volatility to relax the i.i.d. restriction on the spot return dynamics under the baseline models. Tractability in deriving the option pricing formula is preserved under the time-changed Lévy process framework, provided that the characteristic functions of the relevant processes are known. For example, with the use of a time-dependent time deformation process, the baseline GH option pricing model can be readily extended to allow for mean-reverting stochastic volatility of spot returns. This extension is detailed in Section ??. As another example, short- and long-range dependence in volatility can be induced by choosing the time deformation process to be a superposition of many time-dependent processes (?), although its empirical performance is yet to be examined in the literature. Generalising this idea further, it is possible to develop option pricing models with multi-factor volatility dynamics, such as the models in ?, and ?. The multi-factor structure provides the necessary flexibility in modelling the underlying risk-neutral spot dynamics. Empirical evidence suggests that they are capable of capturing option stylised facts (e.g., implied volatility surface) more accurately than the conventional models.

? and ? consider a GH model for option pricing. There are several important differences that separate our approach from theirs. First, using time series data of stock returns, ? and ? estimate the GH model, which is then used to price the options and obtain the model-implied volatilities. This is made possible by means of Esscher transform, which allows them to recover the risk-neutral spot price dynamics from the statistical measure. We take a more direct route by specifying the spot price dynamics under the risk-neutral measure. The risk-neutral parameters of our flexible GH model are then estimated directly using option data. Our modelling approach is therefore in line with the risk-neutral option pricing literature (e.g., ?, ?, ?, ?, and ?). Second, ? and ? express the European option price in terms of the integral of a $\tau$-fold convolution of the GH densities $(\tau$ is the time-to-maturity) which cannot be further simplified as the GH distribution is not closed under convolution. To circumvent the numerical challenge, they employ Fourier inversion to obtain the cumulative probabilities from the characteristic function of the log-return process. By contrast, using the ? approach, we obtain an explicit option pricing formula in terms of the characteristic function of the logarithmic spot price process. Third, in terms of numerical optimisation, ? and ? estimate the risk-neural parameters by first keeping the index parameter fixed and then optimising over the other parameters ${ }^{3}$ We are able to overcome the numerical difficulty by estimating all four parameters of the flexible GH model jointly and efficiently. This is the combined result of a prudent choice of model parameterisation, appropriate variable transformation, and a larger sample offered by the option panel compared to the time series of spot prices.

In addition, we conduct an empirical study of the flexible GH model and its special cases by investigating its performance in modelling S\&P 500 Index options. For a fair comparison, the GH model is evaluated against benchmark option pricing models that assume i.i.d. spot returns, including the finite moment log-stable (FMLS) and Black-Scholes models 4 Among the special cases of GH, we will also evaluate the NIG model, and closely

\footnotetext{
${ }^{1}$ The pure-jump and infinite activity property of the GH process and special cases is a distinguishing property from Heston's model (?), the Black-Scholes model and from other seminal models, such as Merton's jump-diffusion model (?), Bates' model (?), and Pan's model (?), as noted by ?. As a purely discontinuous process, the GH process is also different from the GH diffusion process of ? and ?.

${ }^{2}$ The use of time deformation processes that induce persistent variations in drift and volatility is supported by a general equilibrium argument. For example, see?. We thank one referee who brings this literature to our attention.

${ }^{3}$ Admitting the computational difficulty in estimating the four parameters jointly, ? only presents the risk-neutral estimation results for the NIG model, a special case of the GH option pricing model obtained by fixing the index parameter (or shape parameter according to ?) to $p=-1 / 2$ (see Table 2.28 and the discussion on p.61 of? for details). Nevertheless, ? and ? estimate the GH model parameters under the physical measure using spot data.

${ }^{4}$ The FMLS model of ? features a heavy-tailed, negatively skewed log-return distribution. While not a time-changed Lèvy process model, the FMLS model is chosen for our empirical study over the log-stable model of ? because the former guarantees finite moments of the spot price and hence finite option prices.
} 
examine the VG and $t$ models, which ? and ? precluded from being estimated under the risk-neutral setting. A direct comparison between the VG and $t$ models is theoretically and practically motivated, since the models are complementary limiting cases of the flexible GH model (see Section ??), yet the VG model is much more widely used than the $t$ model in empirical option pricing. The main empirical findings of our paper are summarised as follows. First, among all six models under consideration, the flexible GH model yields the best in-sample fitting and out-of-sample forecast across the entire panel of option prices; the added flexibility offered by the extra shape parameter is indispensable for the superior performance. Second, the $t$ model often performs as good as, and sometimes even better than, the flexible GH model in fitting and predicting the prices of certain option categories. For example, the $t$ model often provides an adequate fit to the left tail of the log-return distribution, as revealed by its superior performance in modelling the in-the-money put options in the main estimation. Third, as shown in the robustness analysis, the superior overall out-of-sample performance of the flexible GH model is preserved over a turbulent sample period in 2011, although the category-specific results are mixed - the FMLS model surpasses the GH model in predicting the prices of at-the-money and in-the-money options, thus pointing to the necessity of excess kurtosis in the spot price distribution for accurate option pricing.

In summary, our contributions are threefold. First, we study a flexible GH option pricing model in the timechanged Lévy process framework. Taking this dynamic perspective is fruitful in option pricing as it motivates the way the GH model is parametrised. The flexible GH model encompasses six option pricing models as special cases, including the popular variance gamma (VG) model and some new models which draw upon some familiar distributions, such as the $t$ distribution. Second, we obtain an explicit, semi-closed form option pricing formula under our GH model. The formula is derived using the characteristic function approach (?). Third, we investigate the empirical performance of the GH model and some of its special cases. By comparison with benchmark models such as the FMLS model (?) and the Black-Scholes model, we illustrate the convenience of calibrating the GH model, and its consistent superiority in delivering in-sample fitting and out-of-sample prediction over the panel of S\&P 500 Index options. To our knowledge, this paper provides the first systematic empirical analysis of the GH model and some of its special cases, including the VG, $t$, and NIG models. Although our empirical study focuses on Lévy-based option pricing models that assume i.i.d. spot returns, the empirical results would shed light on future work on evaluating the empirical performance of stochastic volatility (SV) extensions of such models, some of which are developed in Section ??. It would be interesting, for example, to compare the GH-SV model with conventional ? model, whose underlying spot dynamics are characterised by time-changed GH process and time-changed Brownian motion, respectively.

The paper is structured as follows. Section 1 begins with a description of a subordinated process and presents a corresponding option pricing framework. In Section 2, we formulate the flexible GH option pricing model and its six special cases, including the two limiting cases. Section ?? comprises our empirical study. The data description, parameter estimates, in-sample fit, orthogonality test and out-of-sample pricing results are provided therein. In Section ??, we discuss the extension of the GH model to incorporate stochastic volatility. Section ?? concludes the paper.

\section{Time-changed Lévy Processes and Option Pricing}

We focus on a generic option pricing framework in which the log-return process of the underlying asset is driven by a time-changed Lévy process (?, Carr, Geman, Madan and Yor (thereafter ?), ?). Let $L_{t} \equiv L(t)$ denote a Lévy process, and let $T_{t} \equiv T(t)$ be an increasing, positively-valued time deformation process. The resulting time-changed Lévy process is given by

$$
Z_{t} \equiv Z(t)=L(T(t))
$$

The time deformation process $T_{t}$ represents the total elapsed time in a transformed time scale which may reflect the varying rate at which economic and business activities occur.

As will be explored in this paper, this modelling framework offers flexibility in the specification of the Lévy process and the time deformation process. It encompasses many option pricing models in the literature. In particular, the time deformation process $T_{t}$ can be deterministic (e.g., an identity process $T_{t}=t$, as in BlackScholes model), a pure-jump Lévy process with stationary and independent increments (e.g., a gamma process, 
as in ?, or a time-dependent process, possibly driven by the same random process that drives $L_{t}$ (e.g., ?, ?, ?). To preserve the business clock interpretation, we require that $T_{t}$ satisfies the following conditions:

1. $T_{0}=0$,

2. $T_{t}$ is non-negative and increasing with $t$,

3. $E\left[T_{1}\right]=1$.

The third condition is a normalising restriction on $T_{t}$. If $T_{t}$ is a Lévy process, then, by infinite divisibility, this condition implies that $E\left[T_{t}\right]=t$ for all positive time $t$.

To derive the option pricing formula, it is instrumental that we obtain the characteristic function of the time-changed process $Z_{t}$. Defining the characteristic functions of $L_{t}$ and $T_{t}$ by $\phi_{L_{t}}(u)=E\left(e^{i u L_{t}}\right)$ and $\phi_{T_{t}}(u)=$ $E\left(e^{i u T_{t}}\right)$, respectively, we can compute the characteristic function of $Z_{t}$ as follows

$$
\phi_{Z_{t}}(u)=\phi_{T_{t}}\left(-i \phi_{L_{1}}(u)\right)
$$

We assume that $Z_{t}$ drives the log-return process of the underlying asset. More specifically, under the riskneutral probability spact ${ }^{5}\left(\Omega, \mathbb{Q},\left\{\mathcal{F}_{t}\right\}\right)$ equipped with the filtration $\left\{\mathcal{F}_{t}\right\}$, the spot price of the underlying asset evolves according to the following dynamics: for all $t \geq 0$,

$$
S_{t}=S_{0} \exp \left\{(r-q+\omega) t+Z_{t}\right\}
$$

For simplicity, the risk-free rate $r$ and the dividend yield $q$ are assumed to be constant in our analysis. The drift adjustment $\omega$ is required to ensure that the discounted spot price process is a martingale under $\mathbb{Q}$. It is given by $\omega=-\frac{1}{t} \log \phi_{Z_{t}}(-i)=-\log \phi_{Z_{1}}(-i)$ (see Appendix A). As a result, the characteristic function of the log-price is given by

$$
\phi_{\log S_{t}}(u)=S_{0}^{i u} \exp \{i u(r-q+\omega) t\} \phi_{Z_{t}}(u)
$$

Using the characteristic function approach to option pricing (e.g., ?, ?, ?), the price of a European option written on this underlying asset with the spot price process, defined in (3), can be obtained. The time- $t$ price of a European call with strike price $K$ and time-to-maturity $\tau=T-t$ is

$$
\begin{aligned}
C_{t} & =E^{\mathbb{Q}}\left[e^{-r \tau} \max \left(S_{T}-K, 0\right) \mid \mathcal{F}_{t}\right] \\
& =S_{t} e^{-q \tau} \Pi_{1}-K e^{-r \tau} \Pi_{2},
\end{aligned}
$$

where $E^{\mathbb{Q}}[\cdot]$ denotes the expectation taken under $\mathbb{Q}$, and the quantities $\Pi_{1}$ and $\Pi_{2}$ are the prices of some ArrowDebreu securities (?), given by

$$
\begin{aligned}
& \Pi_{1}=\frac{1}{2}+\frac{1}{\pi} \int_{0}^{\infty} \operatorname{Re}\left(\frac{e^{-i \omega \log (K)} \phi_{\log S_{t}}(\omega-i)}{i \omega \phi_{\log S_{t}}(-i)}\right) d \omega, \\
& \Pi_{2}=\frac{1}{2}+\frac{1}{\pi} \int_{0}^{\infty} \operatorname{Re}\left(\frac{e^{-i \omega \log (K)} \phi_{\log S_{t}}(\omega)}{i \omega}\right) d \omega .
\end{aligned}
$$

By the put-call parity, the price of the corresponding European put option with the same strike price and maturity date is

$$
P_{t}=K e^{-r \tau}\left(1-\Pi_{2}\right)-S_{t} e^{-q \tau}\left(1-\Pi_{1}\right) .
$$

\section{The Baseline Model}

In this section, we introduce the baseline generalised hyperbolic (GH) option pricing model. Recall from (3) that the spot price is driven by the time-changed Lévy process $Z_{t}$. Under the flexible GH model specification,

\footnotetext{
${ }^{5}$ In general, when there are a finite number of assets in the market, the risk-neutral probability measure $\mathbb{Q}$ may not be unique. When the price of the underlying asset exhibits jumps, it typically requires infinite many assets to complete the market (an exception is when the asset price contains Poisson jumps with a finite number of different jump sizes). On the technical level, sufficient and necessary conditions for market completeness are known for a general Lévy process (?). More recently, ? provide a set of sufficient conditions for equivalent measure changes for a subordinated diffusion model.
} 
the time-changed Lévy process $Z_{t}$ is constructed by subordinating a Brownian motion with drift, $B(t)$, to a generalised inverse Gaussian (GIG) process $g(t)$. More precisely, the time-changed Lévy process is defined by

$$
Z_{t}:=Z(t)=B(g(t))=\theta g(t)+\sigma W(g(t))
$$

where $\theta$ is the drift parameter, $\sigma>0$ is the volatility parameter, and $W(t)$ is the standard Wiener process.

The GIG process $g(t)$ is a positively-valued Lévy process such that $g_{1}:=g(1)$ follows the GIG distribution with parameters $p, \gamma$ and $\delta$. The probability density function and the first four moments of the GIG distribution are provided in the Appendix. In particular, by defining the parameter $\zeta:=\gamma \delta$, the mean is

$$
E\left[g_{1}\right]=\frac{\zeta K_{p+1}(\zeta)}{\gamma^{2} K_{p}(\zeta)}
$$

where $K_{j}(\cdot)$ is the modified Bessel function of the third kind with index $j(?)$. The mean of $g_{1}$ is well defined provided that the parameters $p$ and $\zeta$ satisfy the conditions:

(i) $\zeta>0$ if $-1 \leq p \leq 0$,

(ii) $\zeta \geq 0$ otherwise.

By imposing the normalisation $E\left[g_{1}\right]=1$, we obtain the parameter restriction

$$
\gamma^{2}=\zeta \frac{K_{p+1}(\zeta)}{K_{p}(\zeta)}
$$

and hence the GIG distribution is characterised by two parameters: the index parameter $p$ and the shape parameter $\zeta$.

From a static point of view, the process at time $1, Z_{1}$, follows the GH distribution, which can be viewed as a normal mean-variance mixture distribution with a GIG mixing density. For any fixed $t$, the distribution of $Z_{t}$ is skewed and leptokurtic, due to the mixing in the mean through the Brownian motion's drift $\theta$.

From a dynamic point of view, the time-changed Lévy process $Z_{t}$ is constructed by subordinating a drifted Brownian motion to a GIG process. The distribution of $g(t)$ is obtained as the $t$-fold convolution of the GIG distribution of $g_{1}$. In particular, for any $t \geq 0$, the increment $g(t+1)-g(t)$ follows the same distribution as that of $g_{1}$. Note that $g(t)$ is not distributed as GIG for $t \neq 1$, as the GIG distribution is not preserved under convolution. It follows from this dynamic construction that the marginal distribution of $Z_{t}$ is not GH in general except at $t=1$. Nevertheless, by infinite divisibility of the GIG distribution (?), the GIG process $g(t)$ is infinitely divisible. As a result, the time-changed process $Z_{t}$ is a Lévy process, and so it is infinitely divisible and has stationary and independent increments. For a Lévy process, the characteristic functions of $Z_{t}$ and $Z_{1}$ are related by

$$
\phi_{Z_{t}}(u)=\left[\phi_{Z_{1}}(u)\right]^{t}
$$

(e.g., ?).

The central moments of $Z_{t}$ grow over time just like any generic Lévy process. The mean, variance and the third central moment of $Z_{t}$ increase linearly with $t$. In terms of standardised central moments, the skewnesses of $Z_{t}$ and $Z_{1}$ are related by skew $\left(Z_{t}\right)=(1 / \sqrt{t}) \operatorname{skew}\left(Z_{1}\right)$ for $t>0$, and the kurtosis of $Z_{t}$ is a weighted average of the kurtosis of $Z_{1}$ and that of a normal distribution, i.e., $\operatorname{kur}\left(Z_{t}\right)=(1 / t) k u r\left(Z_{1}\right)+3(1-1 / t)$ for $t>0$. Given that $Z_{1}$ follows a GH distribution, we have $\operatorname{skew}\left(Z_{1}\right) \neq 0$ if the drift parameter $\theta$ is non-zero, and $k u r\left(Z_{1}\right)>3$. The same moment properties are therefore valid for $Z_{t}$ for all $t>0$. A study of the moment properties is detailed in Appendix B.

To price a European option under the flexible GH model, it is necessary to get the characteristic function of $Z_{t}$. Using the characteristic function of the GH distribution (see Appendix B), we obtain

$$
\phi_{Z_{t}}(u)=\left[1-\frac{2}{\gamma^{2}}\left(i u \theta-\frac{1}{2} \sigma^{2} u^{2}\right)\right]^{\frac{-p t}{2}}\left[\frac{K_{p}\left(\zeta \sqrt{1-\frac{2}{\gamma^{2}}\left(i u \theta-\frac{1}{2} \sigma^{2} u^{2}\right)}\right)}{K_{p}(\zeta)}\right]^{t} .
$$


Provided that $\theta<\frac{\gamma^{2}-\sigma^{2}}{2}$, the drift adjustment is well-defined and is given by

$$
\omega=-\log \phi_{Z_{1}}(-i)=-\frac{p}{2} \log \left[1-\frac{2}{\gamma^{2}}\left(\theta+\frac{\sigma^{2}}{2}\right)\right]+\log \left[\frac{K_{p}\left(\zeta \sqrt{1-\frac{2}{\gamma^{2}}\left(\theta+\frac{\sigma^{2}}{2}\right)}\right)}{K_{p}(\zeta)}\right]
$$

The European option under the flexible GH model can then be priced using (??) and (??).

\subsection{Special Cases of the Flexible GH Model}

In the family of risk-neutral spot price models with independent and stationary increments, the baseline GH option pricing model is quite flexible in that it encompasses a number of particular cases. In this section, we study six of its special cases, all of which are indexed by three parameters. These six models can be classified into two groups: four special cases obtained by restricting the value of the index parameter $p$, while the other two obtained as limiting cases as the shape parameter $\zeta:=\delta \gamma \rightarrow 0$. Table ?? summarises the parameter restrictions required to obtain the six special cases of the flexible $\mathrm{GH}$ model.

The first group of three-parameter sub-models is obtained as follows. By setting $p=1$, the flexible GH model reduces to a hyperbolic $(\mathrm{H})$ model, in which the mixing random variable $g_{1}$ follows the positive hyperbolic mixture distribution. Setting $p=-1$ leads to a reciprocal hyperbolic $(\mathrm{RH})$ model, in which the mixing distribution is reciprocal positive hyperbolic. For $p=-\frac{1}{2}$, we obtain the normal inverse Gaussian (NIG) distribution, generated by a normal mixture of the inverse Gaussian distribution. For $p=\frac{1}{2}$, we obtain the normal reciprocal inverse Gaussian (NRIG) distribution, generated by a normal mixture of the reciprocal inverse Gaussian distribution.

The second group of sub-models is obtained by restricting the shape parameter $\zeta$ to 0 and the sign of the index parameter $p$. By letting $\delta \rightarrow 0$ and restricting $p>0$, we obtain the VG model (Madan, Carr and Chang (1998)), in which $g_{1}$ follows the gamma distribution with parameters $p$ and $\frac{\gamma^{2}}{2}$. By letting $\gamma \rightarrow 0$ and restricting $p<0$, we obtain the $t$ model, in which $g_{1}$ follows the reciprocal (or inverse) gamma distribution with parameters $-p$ and $\frac{\delta^{2}}{2}$. With the normalisation condition $E\left[g_{1}\right]=1$ in place, we deduce that $\gamma=\sqrt{2 p}$ in the VG case and $\delta=\sqrt{2(-p-1)}$ in the $t$ case.

Figure ?? demonstrates the flexibility of the GH model in the modelling of option prices relative to its six special cases. The plotted surface represents the prices of an in-the-money call option under the GH model for varying $p$ and $\zeta$ (the drift and volatility parameters are fixed). The option prices associated with the first group of sub-models (H, NRIG, NIG and RH models) are obtained on the four dotted cross-sections of the surface at the respective fixed values of $p$, while those associated with the second group of sub-models (VG and $t$ models) are found respectively along the positive and negative sections of the $p$ axis at the boundary $\zeta=0$. When both the index and shape parameters are close to zero, the GH model implies a high kurtosis in the risk-neutral distribution of spot prices. This translates into more extreme option prices (lower prices under NIG, RH, and $t$ with $p$ close to 0 , and higher prices under NRIG, H, and VG with $p$ close to 0 ). The GH model offers the most versatility as manifested by the wide range of option prices it generates over all possible combinations of the index and shape parameters. 
Table 1: Special cases of the GH option pricing model.

\begin{tabular}{|c|c|c|}
\hline $\mathbf{G H}(p, \zeta, \theta, \sigma)$ parameter conditions & Density of $X_{1}$ & Density of $g_{1}$ \\
\hline Hyperbolic model & & Positive hyperbolic \\
\hline $\operatorname{GH}(1, \zeta \geq 0, \theta, \sigma), \delta \geq 0, \gamma>0$ & $\mathrm{H}(\zeta, \theta, \sigma)$ & $\operatorname{PH}(\delta, \gamma)$ \\
\hline Reciprocal hyperbolic model & & Reciprocal positive hyperbolic \\
\hline $\mathrm{GH}(-1, \zeta>0, \theta, \sigma), \delta>0, \gamma>0$ & $\mathrm{RH}(\zeta, \theta, \sigma)$ & $\operatorname{RPH}(\delta, \gamma)$ \\
\hline Normal inverse Gaussian model & & Inverse Gaussian \\
\hline $\mathrm{GH}\left(-\frac{1}{2}, \zeta>0, \theta, \sigma\right), \delta>0, \gamma>0$ & $\operatorname{NIG}(\zeta, \theta, \sigma)$ & $\operatorname{IG}(\delta, \gamma)$ \\
\hline Normal reciprocal inverse Gaussian model & & Reciprocal inverse Gaussian \\
\hline $\mathrm{GH}\left(\frac{1}{2}, \zeta \geq 0, \theta, \sigma\right), \delta \geq 0, \gamma>0$ & $\operatorname{NRIG}(\zeta, \theta, \sigma)$ & $\operatorname{RIG}(\delta, \gamma)$ \\
\hline Variance gamma model & & Gamma \\
\hline $\mathrm{GH}(p>0,0, \theta, \sigma), \delta=0, \gamma=\sqrt{2 p}$ & $\operatorname{VG}(p, \theta, \sigma)$ & $\Gamma(p, p)$ \\
\hline$t$ model & & Reciprocal gamma \\
\hline $\mathrm{GH}(p<-1,0, \theta, \sigma), \delta=\sqrt{2(-p-1)}, \gamma=0$ & $t_{-2 p}(-p, \theta, \sigma)$ & $\mathrm{R} \Gamma(-p,-p-1)$ \\
\hline
\end{tabular}

$\zeta=\gamma \delta$. For the hyperbolic, reciprocal hyperbolic, normal inverse Gaussian and normal reciprocal inverse Gaussian models, $\gamma$ is given by Equation (5). For all models, $\mathrm{E}\left[g_{1}\right]=1$.

Figure 1: Simulated option prices under the GH model.

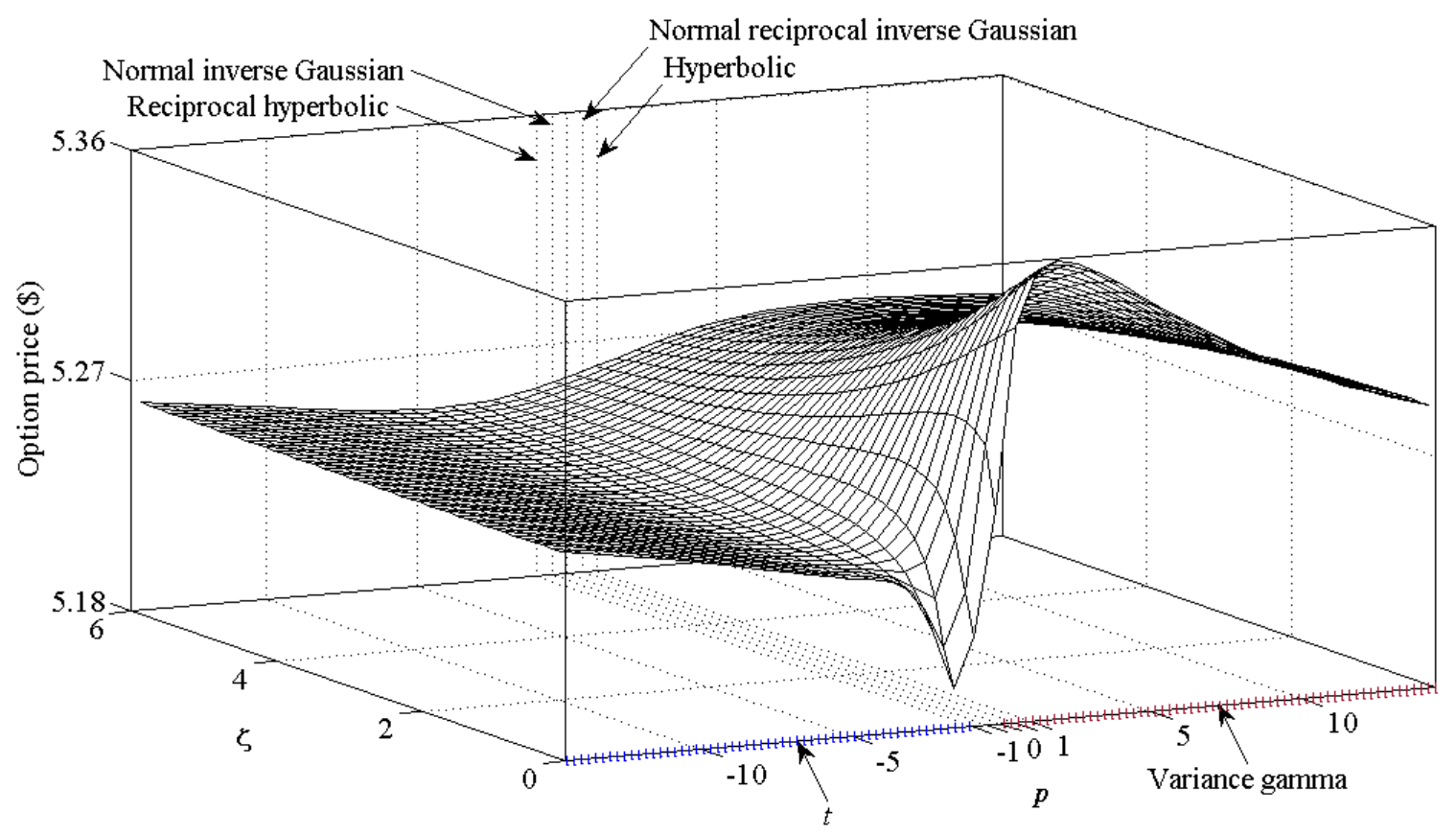

The price of an in-the-money call option with a strike price equal to $95 \%$ of the spot price, time-tomaturity equal to 3 weeks, $\theta=-0.04$ and $\sigma=0.17$. The six special cases are reciprocal hyperbolic at $p=-1$, normal inverse Gaussian at $p=-\frac{1}{2}$, normal reciprocal inverse Gaussian at $p=\frac{1}{2}$, hyperbolic at $p=1$, variance gamma at $\zeta=0$ and $p>0$, and the $t$ model at $\zeta=0$ and $p<-1$. At $\zeta=0$ and $-1 \leq p \leq 0$, the mean of the GH distribution is undefined. 


\subsection{The VG Model}

The characteristic function of the logarithmic spot price under the VG model is given by

$$
\phi_{\log S_{t}}(u)=S_{0}^{i u} \exp \{i u(r-q+\omega) t\}\left[1-\nu\left(i u \theta-\frac{1}{2} \sigma^{2} u^{2}\right)\right]^{-\frac{t}{\nu}}
$$

where $\omega=\frac{1}{\nu} \log \left[1-\nu\left(\theta+\frac{1}{2} \sigma^{2}\right)\right]$ for $\theta<\left(\frac{1}{\nu}-\frac{\sigma^{2}}{2}\right)$. This formula can be obtained as the limit of the corresponding characteristic function under the GH model (see Appendix C). This is equivalent to the VG option pricing model of Madan, Carr and Chang (1998), which contains three parameters: the drift $\theta$, the volatility $\sigma$, and the mixing distribution variance $\nu$ (which is equal to $\frac{1}{p}$ using our index parameter). The distributional properties of the VG model are examined in Appendix B.2.

\subsection{The $t$ Model}

Let us turn to the $t$ option pricing model ${ }^{6}$ Under this model, the log-return of the underlying asset follows a skewed $t$ distribution with $-2 p$ degrees of freedom. The characteristic function of the logarithmic spot price under the VG model is given by

$$
\begin{aligned}
\phi_{\log S_{t}}(u) & =S_{0}^{i u} \exp \{i u(r-q+\omega) t\} \times \\
& {\left[\frac{2\left(\frac{1}{\nu}-1\right)^{\frac{1}{2 \nu}}}{\Gamma\left(\frac{1}{\nu}\right)}\left[-\left(i u \theta-\frac{1}{2} \sigma^{2} u^{2}\right)\right]^{\frac{1}{2 \nu}} K_{\frac{1}{\nu}}\left(\sqrt{-4\left(\frac{1}{\nu}-1\right)\left(i u \theta-\frac{1}{2} \sigma^{2} u^{2}\right)}\right)\right]^{t}, }
\end{aligned}
$$

where $\omega=-\log \left[\frac{2\left(\frac{1}{\nu}-1\right)^{\frac{1}{2 \nu}}}{\Gamma\left(\frac{1}{\nu}\right)}\left[-\left(\theta+\frac{1}{2} \sigma^{2}\right)\right]^{\frac{1}{2 \nu}} K_{\frac{1}{\nu}}\left(\sqrt{-4\left(\frac{1}{\nu}-1\right)\left(\theta+\frac{1}{2} \sigma^{2}\right)}\right)\right]$ for $\theta<-\frac{\sigma^{2}}{2}$, and $K_{j}(\cdot)$ is the modified Bessel function of the third kind with index $j$. This formula can be obtained as the limit of the corresponding characteristic function under the flexible GH model (see Appendix C). The spot price dynamics under the $t$ model is equivalent to the GH skewed-student's $t$ model in ? after re-parameterisation, except that the latter specifies the spot price process under the statistical measure and is not used for option pricing. The distributional properties of the $t$ model are examined in Appendix B.3.

\section{Empirical Study}

\subsection{The Data}

The data used for our main estimation are the daily prices of S\&P 500 Index European options. Put options are featured in our sample because, compared to their call counterparts, their prices carry more information about the left tail of the price distribution, corresponding to the side to which the price distribution is found to be skewed empirically (?).

The sample period is chosen to be 2012 with 250 trading days. It contains some moderate upward and downward trends in the S\&P 500 Index. The volatility index (VIX) fluctuates mildly between 13 and 27 percent over the period. Both series are displayed in the bottom two panels in Figure ??. In the robustness analysis in Section ??, we will choose the most turbulent period in 2011 as our alternative sample and repeat the analysis.

The cross-sectional data structure for the put option sample used in the main estimation is summarised in Table ??. To retain a majority of put options that are more liquidly traded in the market, we focus on the puts whose moneyness (the strike-to-spot price ratio) lies between 0.94 and 1.06, and whose time-to-maturity is longer than a week. On each day, the sample retains only the three batches of put options belonging to the short-term, medium-term and long-term categories, corresponding to the nearest-, second-nearest-, and thirdnearest-to-maturity put options, respectively. This leaves us with 60 put options on average per day. In total, the option panel consists of 15,058 put prices. To price the European puts, we use the annualised yield of the

\footnotetext{
${ }^{6}$ A similar version of the $t$ option pricing model was proposed in Yeap (2014), except that a different parameterisation $\nu=\operatorname{Var}\left(g_{1}\right)$ was adopted. In this paper, we enforce the normalisation $E\left(g_{1}\right)=1$ so that $\operatorname{Var}\left(g_{1}\right)=\frac{\nu}{1-2 \nu}$. Our model allows for skewness in the log-return distribution, whereas a symmetric $t$ option pricing model has been proposed (?).
} 
one-month U.S. Treasury bill as the risk-free rate. The time series of S\&P 500 Index is adjusted by the mean annualised dividend yield of the stocks.

Due to the way the sample is selected, the time-to-maturities exhibit some artificial cyclicity over time. This can be seen from the time series plot of the average time-to-maturity at the bottom panel of Figure ??. Note that the S\&P 500 Index options expire on the third Friday of each month. As the maturity date approaches, the time-to-maturities of the options in the sample decreases gradually over time until some of them (i.e., the time-to-maturities of those puts in the short-term category) falls below one week, at which point these nearestto-maturity options will be excluded from the sample and replaced by a new batch of options that forms the long-term category. The original long-term (medium-term) category now becomes the medium-term (short-term) category. This roll-over of the sample, which occurs around the second Friday of each month, leads to an abrupt increase in the time-to-maturities by approximately one month across the three term categories. It is important to bear this in mind when we interpret the time series variation of the parameter estimates.

Table 2: Options data characteristics of the main estimation sample.

\begin{tabular}{lcccc}
\hline & $\begin{array}{c}\text { Short-term } \\
1 \text { week }<\tau<1 \text { month }\end{array}$ & $\begin{array}{c}\text { Medium-term } \\
1 \text { month } \leq \tau \leq 3 \text { months }\end{array}$ & $\begin{array}{c}\text { Long-term } \\
\tau>3 \text { months }\end{array}$ & All \\
\hline Out-of-the-money & $8 \%$ & $24 \%$ & $11 \%$ & $43 \%$ \\
$0.94<\frac{K}{S}<0.98$ & {$[\$ 3.49]$} & {$[\$ 13.40]$} & {$[\$ 26.86]$} & {$[\$ 14.87]$} \\
At-the-money & $8 \%$ & $22 \%$ & $11 \%$ & $41 \%$ \\
$0.98 \leq \frac{K}{S} \leq 1.02$ & {$[\$ 16.53]$} & {$[\$ 29.20]$} & {$[\$ 43.80]$} & {$[\$ 30.55]$} \\
In-the-money & $4 \%$ & $9 \%$ & $3 \%$ & $16 \%$ \\
$1.02<\frac{K}{S}<1.06$ & {$[\$ 51.81]$} & {$[\$ 58.79]$} & {$[\$ 71.32]$} & {$[\$ 59.01]$} \\
All & $20 \%$ & $55 \%$ & $25 \%$ & $100 \%$ \\
& {$[\$ 18.29]$} & {$[\$ 27.48]$} & {$[\$ 39.17]$} & {$[\$ 28.42]$} \\
\hline
\end{tabular}

A contingency table for the sample of S\&P 500 Index European put option prices in 2012. The sample is selected according to the criteria in the Data section. $n=15,058$. Average option prices are displayed in square brackets. $K$ is the strike price, $S$ is the spot price, and $\tau$ is the time-to-maturity.

\subsection{Model Calibration and Parameter Estimate}

The option pricing models are calibrated daily by minimizing the sum of squared percentage pricing errors $S S P E_{t}(\Theta)$ on each date $t$ :

$$
S S P E_{t}(\Theta)=\sum_{i=1}^{n_{t}}\left(\frac{\hat{O}_{i t}(\Theta)-O_{i t}}{O_{i t}}\right)^{2},
$$

where $\Theta$ is the vector of model parameters, $n_{t}$ is the cross-sectional sample size on date $t$, and $\hat{O}_{i t}(\Theta)$ and $O_{i t}$ are the predicted and observed option prices (in dollars), respectively. The optimisation search for $\Theta$ is carried out over the parameter space using the Nelder-Mead simplex algorithm. It is implemented through the MATLAB function fminsearch. To mitigate the sensitivity of the solution on the initial value of the parameter vector, a preliminary search is conducted as follows: starting with many different candidate vectors for $\Theta^{[0]}$, we run a first-stage optimisation with a fixed number of iterations (e.g., 10), then we select the optimal candidate vector that yields the smallest $S S P E_{t}(\Theta)$. Starting at this optimal candidate vector, the numerical algorithm is then run until the local minimum is found.

\subsection{In-Sample Estimation}

In this section, we present the main estimation results and examine the in-sample fit of various models. Table ?? summarises the risk-neutral parameter estimates under the Black-Scholes model (BS), the finite-moment log-stable model (FMLS), as well as the GH model and three of its special cases (NIG, VG and $t$ ).

Starting with the volatility parameter $\sigma$, which appears in all models being considered, the FMLS model has the smallest average equal to $9.9 \%$, followed by the BS model (14.6\%), the VG model (15.0\%), the NIG model 
(15.2\%), and then the $t$ and GH models (both 15.8\%). The $t$ model yields the single largest estimate equal to $24.8 \%$.

The skewness parameter $\theta$ is contained in all models except for BS and FMLS7 It is estimated to be negative on average with a similar value across models: -0.044 for the NIG and VG models, -0.047 for the $t$ model, and -0.046 for the GH model. However, the most extreme estimate is obtained under the $t$ model: -0.149 , compared to -0.106 for the NIG model, -0.100 for the VG model and -0.098 for the GH model.

The time series plots of the daily estimates of $\sigma$ and $\theta$ under the GH model are displayed in the top two panels of Figure ??. They reveal a negative association between the volatility and skewness parameters of the log-return distribution, confirming the presence of leverage effect (?): the volatility tends to be higher when the log-returns are more negatively skewed.

Let us turn to the shape parameters ( $\alpha$ for FMLS, and $p$ and $\zeta$ for GH and its special cases). Under the FMLS model, the estimates of the tail index $\alpha$ lie in a narrow range between 1.848 and 1.999, with an average estimate equal to 1.927. By the properties of the asymmetric stable distribution (Property 1.5 of ?), the logreturn distribution has infinite moments of order 2 and higher if $\alpha<2$. Under the GH model and its special cases, the shape is controlled by $p$ (the index parameter) and $\zeta$. Recall that $p$ is constrained to be positive under the VG model, negative under the $t$ model, and equal to -0.5 under the NIG model, and that $\zeta \rightarrow 0$ under the VG and $t$ models, but $\zeta$ is free under the NIG model. The smaller the magnitude of $p$ and $\zeta$, the fatter the tail of the log-return distribution. From the empirical estimation, the VG model yields an average estimate of $p$ equal to 9.305 , which is higher in magnitude than that under the $t$ model $(-5.550$, which translates into 11.1 degrees of freedom). The $p$ estimates under the GH model average to -5.151 and range over -30.180 to 8.014 , with a minimum magnitude of 1.117. These estimates are more in line with the $t$ model than to the VG model. The estimation of $p$ under the flexible GH model indicates that the special cases of GH, including RH $(p=-1)$, NIG $\left(p=-\frac{1}{2}\right)$, NRIG $\left(p=\frac{1}{2}\right)$ and $\mathrm{H}(p=1)$, are not supported by the data. In terms of the shape parameter $\zeta$, the average estimate is 4.910 under the NIG model, but the average is only 0.417 under the flexible GH model. The discrepancy indicates that, due to the restriction on $p, \zeta$ needs to be inflated substantially under the NIG model in order to capture the excess kurtosis of the risk-neutral distribution. This highlights the importance of allowing the index parameter $p$ to be free. The time series of daily estimates of $p$ under the various GH sub-models are plotted in Figure ??. It reveals a cyclical behaviour in the degree of tail-thickness in the log-return distribution that aligns with the option expiration schedule 8 The tail of log-return distribution starts off thick at the start of the trading month (low magnitude of $p$ ), and gradually become thinner (increasing magnitude of $p$ ) towards the second Friday of each month. Then, drastically, the index parameter $p$ falls in magnitude and the log-return distribution becomes fat-tailed again. This pattern repeats itself every month from there on.

\footnotetext{
${ }^{7}$ The log-returns (net of the risk-free rate and dividend yield) follow a symmetric normal distribution under BS and a negatively-skewed stable distribution under the FMLS model (with skewness parameter -1 ).

${ }^{8}$ Alternatively, we may smooth out the cyclicity by using a dynamically weighted sample in which different weights are assigned to options with different time-to-maturities. The weights are determined such that the average time-to-maturity of the weighted sample is maintained at a constant level. The advantage of our current method (by retaining the raw data) is that the term structure of the risk-neutral distribution, and hence the relationship between the shape parameters and the time-to-maturity, becomes more apparent.
} 
Table 3: Summary statistics of risk-neutral parameter estimates (in-sample).

\begin{tabular}{|c|c|c|c|c|}
\hline Parameter & Mean & Standard deviation & Minimum & Maximum \\
\hline \multicolumn{5}{|c|}{ Black-Scholes } \\
\hline$\sigma$ & 0.146 & 0.017 & 0.116 & 0.206 \\
\hline RMSPE & $16.69 \%$ & $4.49 \%$ & $8.85 \%$ & $34.65 \%$ \\
\hline \multicolumn{5}{|c|}{ FMLS model } \\
\hline$\sigma$ & 0.099 & 0.013 & 0.076 & 0.142 \\
\hline$\alpha$ & 1.927 & 0.040 & 1.848 & 1.999 \\
\hline RMSPE & $12.00 \%$ & $5.18 \%$ & $4.81 \%$ & $30.63 \%$ \\
\hline \multicolumn{5}{|l|}{ NIG model } \\
\hline$\sigma$ & 0.155 & 0.022 & 0.121 & 0.231 \\
\hline$\theta$ & -0.044 & 0.019 & -0.108 & -0.007 \\
\hline$\zeta$ & 4.910 & 5.791 & 0.006 & 33.744 \\
\hline RMSPE & $9.10 \%$ & $4.45 \%$ & $3.78 \%$ & $24.24 \%$ \\
\hline \multicolumn{5}{|l|}{ VG model } \\
\hline$\sigma$ & 0.150 & 0.022 & 0.114 & 0.224 \\
\hline$\theta$ & -0.044 & 0.016 & -0.100 & -0.009 \\
\hline$p$ & 9.305 & 9.752 & 1.777 & 62.350 \\
\hline RMSPE & $9.50 \%$ & $3.81 \%$ & $4.42 \%$ & $26.52 \%$ \\
\hline \multicolumn{5}{|l|}{$t$ model } \\
\hline$\sigma$ & 0.158 & 0.025 & 0.113 & 0.248 \\
\hline$\theta$ & -0.047 & 0.021 & -0.149 & -0.013 \\
\hline$p$ & -5.550 & 5.569 & -42.824 & -1.448 \\
\hline RMSPE & $8.38 \%$ & $3.28 \%$ & $3.51 \%$ & $23.98 \%$ \\
\hline \multicolumn{5}{|l|}{ GH model } \\
\hline$\sigma$ & 0.158 & 0.025 & 0.113 & 0.225 \\
\hline$\theta$ & -0.046 & 0.020 & -0.098 & -0.012 \\
\hline$p$ & -5.151 & 5.465 & -30.180 & 8.014 \\
\hline$\zeta$ & 0.417 & 1.483 & 0.000 & 18.778 \\
\hline RMSPE & $8.38 \%$ & $3.27 \%$ & $3.51 \%$ & $23.98 \%$ \\
\hline
\end{tabular}

Parameter estimates and RMSPE (root mean squared percentage error) are obtained daily for the year 2012, which includes 15,058 put prices over 250 days. The average daily sample size is 60 . 
Figure 2: Time series plots of daily risk-neutral volatility $(\sigma)$ and skewness $(\theta)$ parameter estimates under the GH option pricing model, and the plots of the S\&P 500 Index and VIX.
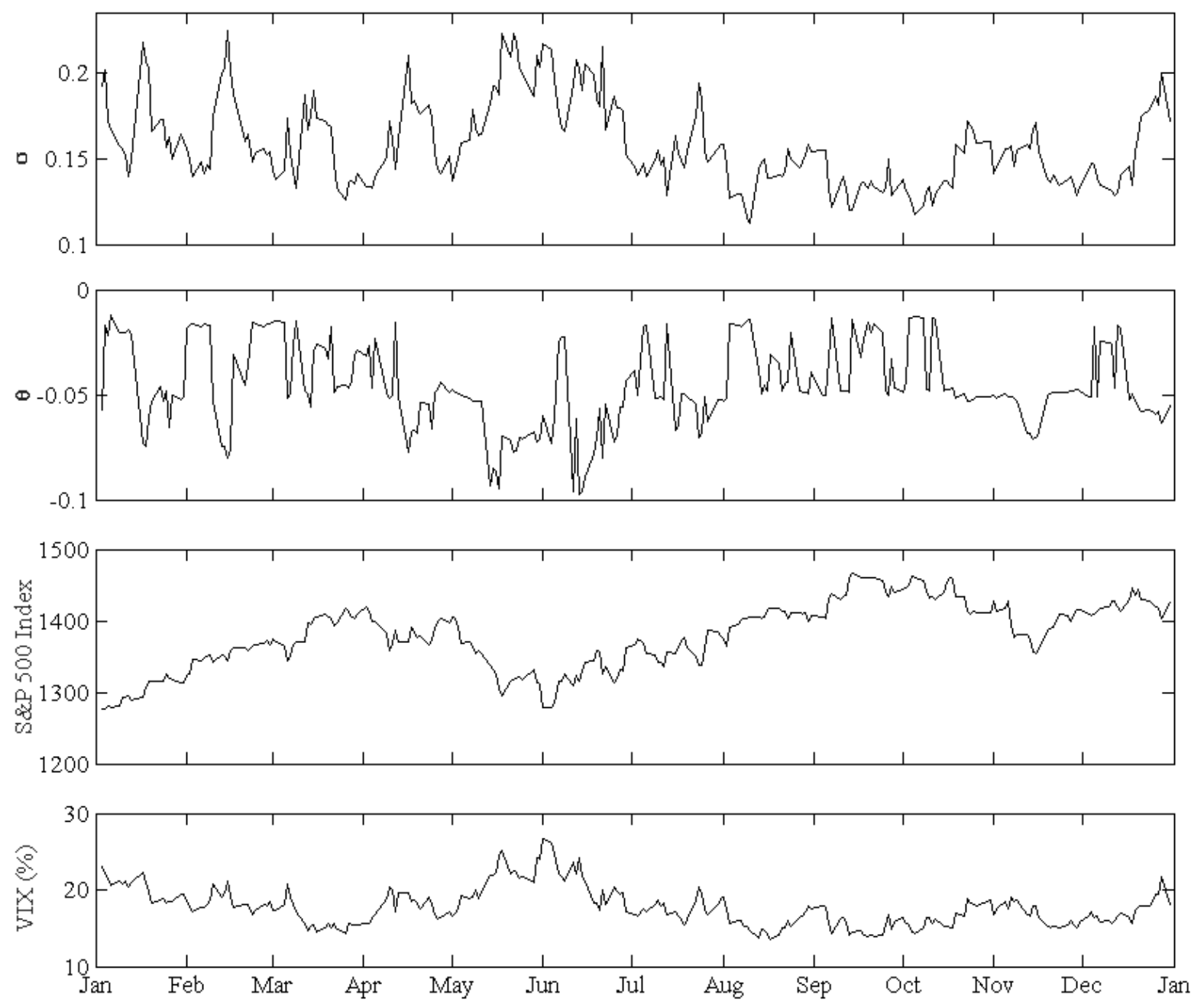

The sample data are the S\&P 500 Index European put prices in 2012, selected according to the criteria detailed in the Data section. The plots include the volatility parameter (top panel), the skewness parameter (second panel), the S\&P 500 Index (third panel), and VIX (bottom panel), the volatility index that measures the implied volatility of S\&P 500 Index options. 
Figure 3: Time series plots of daily risk-neutral shape parameter estimates from different models, and the plot of daily averages of time-to-maturity in the main estimation sample in 2012.
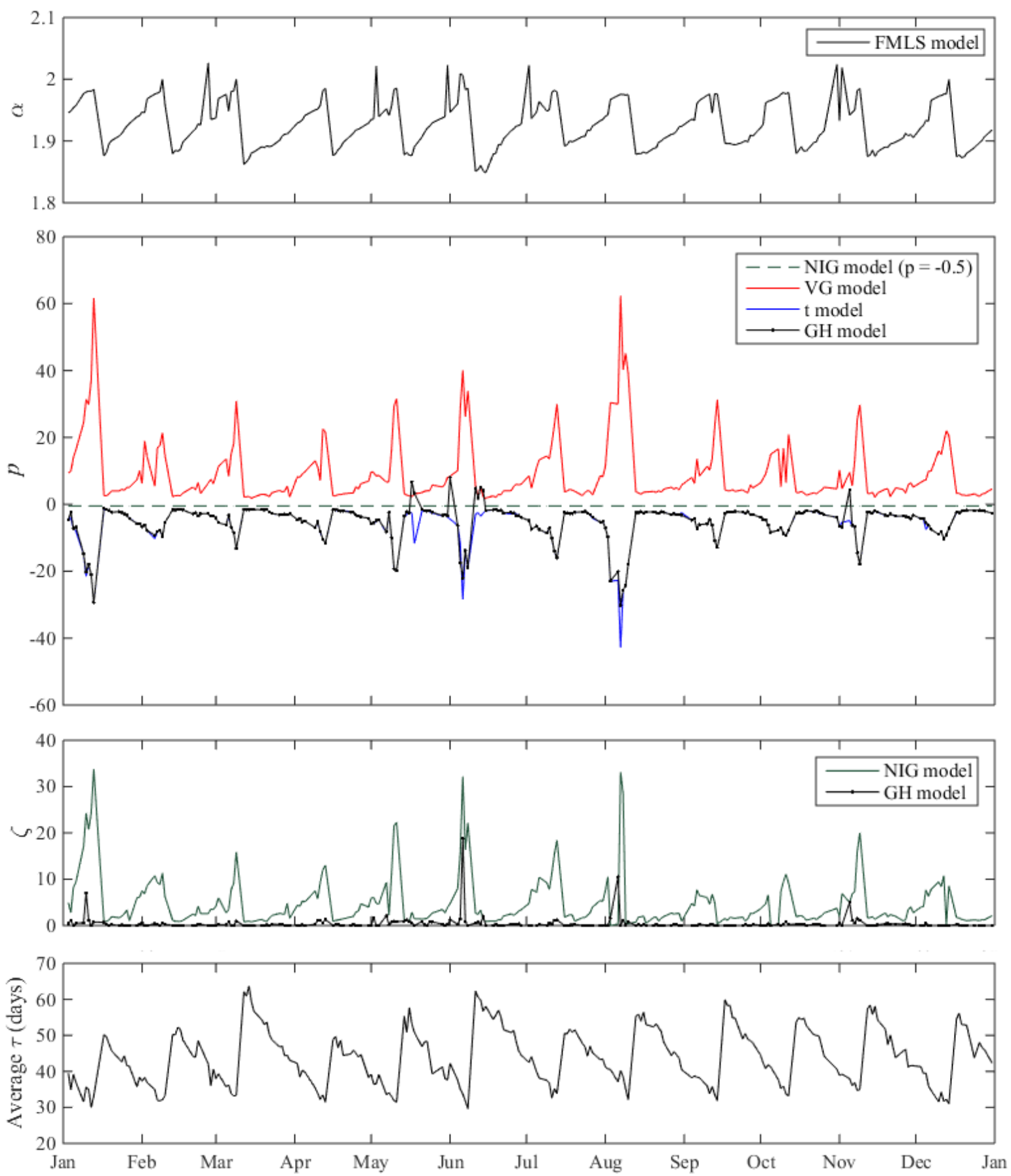

The sample data are the S\&P 500 Index European put prices in 2012, selected according to the criteria detailed in the Data section. The shape parameters include the tail index parameter $\alpha$ under the FMLS model (top panel), and the index parameter $p$ under the GH model and its special cases (second panel), and the shape parameter $\zeta$ defined in Section 2 under the GH and NIG models (third panel), with $\zeta \rightarrow 0$ under the VG and $t$ models. The average time-to-maturity ( $\tau$, in days) is computed by averaging the time-to-maturity of the cross-section of puts each day (bottom panel). 


\subsection{In-Sample Pricing Performance}

We turn now to the first of the performance measures, in-sample pricing error, also reported in Table ??. As expected, the daily-averaged root mean squared percentage error (RMSPE) for the Black-Scholes model is the highest at $16.69 \%$, since it is the most restrictive model with only one parameter controlling the dispersion of the log-return distribution. The FMLS model reduces the RMSPE to $12.00 \%$, the reduction in error representing an improvement to in-sample fit offered by the kurtosis parameter and a fixed negative skewness parameter. The VG, NIG and $t$ models further reduce the RMSPE to $9.50 \%, 9.10 \%$ and $8.38 \%$, respectively. The further improved fit is due to the flexibility in the skewness parameter and the evidently more correct specification of the log-return distribution over the FMLS model.

The better performance of the $t$ model over the VG model indicates that the $t$ distribution is a more plausible model empirically for the risk-neutral log-return distribution 9 The superiority of the $t$ model over other submodels is corroborated by the GH model's parameter estimates: the index parameter $p$ are predominantly negative, and the estimates of the shape parameter $\zeta$ are close to zero. The in-sample fit of the $t$ model, as revealed by the average RMSPE, is virtually as good as that of the GH model (8.38\%).

From the main estimation results, the GH model family clearly outperforms the BS and FMLS models. Allowing $p$ to be free is crucial to the observed improvement in the in-sample fit, although the benefit of having an additional shape parameter $\zeta$ is not fully exploited in this particular dataset. We however wish to emphasise that, while the results are valid for the baseline dataset with sample period spanning 2012, the conclusions may depend on the sample period used. For comparison, the results of the robustness analysis based on a different sample period are presented in Section ??.

\subsection{In-Sample Performance by Option Categories}

While Table ?? offers insight to overall in-sample fit, in Table ?? we examine how the models perform in terms of in-sample fit for different types of put options in the cross section, classified according to different moneyness and time-to-maturity groups as defined in Table ??.

Let us start by comparing the performance across the moneyness categories. For at-the-money (ATM) options, the GH model achieves the best in-sample pricing performance compared to other models: its average RMSPE is $6.78 \%$, compared to $17.15 \%$ for the Black-Scholes model, $11.02 \%$ for the FMLS model, $8.07 \%$ for the VG model, $7.97 \%$ for the NIG model, and $6.79 \%$ for the $t$ model. For in-the-money (ITM) options, the $t$ model offers the best in-sample fit, with an average RMSPE of $8.24 \%$, outperforming the GH model (8.27\%). As all options in our sample are European puts, this suggests that the $t$ distribution provides an optimal fit of the left tail of the log-return distribution. As for out-of-the-money (OTM) options, the GH model ties with the $t$ model, and both of the models clearly outperform the other competitors. Similarly, a comparison across the different time-to-maturity categories reveals that the GH and $t$ models give the best in-sample fit among all models, with the $t$ model comes slightly ahead in pricing the short-dated puts, and the GH model becomes slightly better in pricing the medium-dated puts.

Given that the FMLS model is outside the GH model family, one may be curious whether its peculiar features (e.g., infinite kurtosis of the log-return distribution) would facilitate more accurate pricing of certain options. Table ?? reveals that the FMLS model offers the best fit to the long-dated, ITM puts, achieving the smallest average RMSPE of $9.31 \%$ among all other models. As the price of a long-dated, ITM put is sensitive to the extreme left tail of the distribution of log-returns from now until maturity, the empirical result points to the necessity of excess kurtosis to model the extreme negative returns over a long time horizon. In this sense, the negatively-skewed $\alpha$-stable distribution under the FMLS model provides a better fit than the GH model, in which the kurtosis of log-returns tends to that of a normal distribution as the time horizon increases (see the Appendix). Nevertheless, the FMLS model is not adequate in modelling other sections of the long-range logreturn distribution. Its RMSPE for long-dated OTM puts (14.31\%) is worse than all other models, including the

\footnotetext{
${ }^{9}$ In particular, the empirical skewness and kurtosis of log-returns as implied from the mean parameter estimates under the $t$ model are equal to -0.25 and 4.04 , respectively, which are both bigger in magnitude than those under the VG model (-0.09 and 3.33). The empirical mean and variance are similar under the two models (-0.04 and 0.02 under the VG model; -0.05 and 0.03 under the $t$ model).
} 
BS model (12.73\%). Even though the log-return distribution under the FMLS model has the fattest tails, the empirical fit in the parts other than the left tail is not the best compared to other models with finite kurtosis.

For robustness analysis of the in-sample performance measure, the results for the mean absolute percentage pricing error (MAPE) are also computed. While the average MAPE measures are generally smaller than the average RMSPE measures, probably due to the effect of extreme pricing errors on RMSPE, changing the measure to MAPE retains the optimal model in any given option category.

Table 4: In-sample pricing root mean squared percentage errors (RMSPE) and mean absolute percentage errors (MAPE).

\begin{tabular}{|c|c|c|c|c|c|c|c|c|}
\hline & \multicolumn{4}{|c|}{ In-sample RMSPE (\%) } & \multicolumn{4}{|c|}{ In-sample MAPE (\%) } \\
\hline & \multicolumn{4}{|c|}{ Time-to-maturity } & \multicolumn{4}{|c|}{ Time-to-maturity } \\
\hline & Short & Medium & Long & All & Short & Medium & Long & All \\
\hline \multicolumn{9}{|c|}{ Out-of-the-money } \\
\hline Black-Scholes & 33.03 & 15.26 & 12.73 & 19.57 & 25.78 & 12.02 & 10.12 & 14.23 \\
\hline FMLS model & 26.94 & 12.51 & 14.31 & 16.73 & 20.09 & 9.80 & 11.89 & 12.34 \\
\hline NIG model & 16.33 & 11.14 & $\underline{12.13}$ & 12.57 & 10.35 & 8.45 & 9.47 & 9.08 \\
\hline VG model & 15.72 & 11.31 & 12.71 & 12.64 & 10.98 & 8.55 & 10.01 & 9.39 \\
\hline$t$ model & 10.45 & 10.80 & 12.19 & $\underline{11.10}$ & $\underline{8.06}$ & 8.04 & $\underline{9.46}$ & $\underline{8.40}$ \\
\hline GH model & $\overline{10.47}$ & 10.78 & 12.19 & $\underline{11.10}$ & 8.07 & 8.04 & $\overline{9.46}$ & 8.40 \\
\hline \multicolumn{9}{|c|}{ At-the-money } \\
\hline Black-Scholes & 25.31 & 16.34 & 9.55 & 17.15 & 23.37 & 15.29 & 8.38 & 15.06 \\
\hline FMLS model & 19.13 & 8.53 & 6.24 & 11.02 & 15.77 & 7.31 & 5.13 & 8.40 \\
\hline NIG model & 12.69 & 6.55 & 5.76 & 7.97 & 9.26 & 5.30 & 4.70 & 5.92 \\
\hline VG model & 12.83 & 6.66 & 5.80 & 8.07 & 9.99 & 5.42 & 4.75 & 6.14 \\
\hline$t$ model & 9.63 & $\underline{6.03}$ & 5.56 & 6.79 & 7.93 & $\underline{5.03}$ & 4.51 & 5.46 \\
\hline GH model & $\underline{9.61}$ & $\underline{6.03}$ & $\underline{5.55}$ & 6.78 & 7.91 & $\underline{5.03}$ & 4.50 & $\overline{5.46}$ \\
\hline \multicolumn{9}{|l|}{ In-the-money } \\
\hline Black-Scholes & 7.78 & 12.29 & 12.47 & 11.34 & 6.57 & 11.77 & 12.20 & 10.51 \\
\hline FMLS model & 6.85 & 9.03 & $\underline{9.31}$ & 8.57 & 5.90 & 8.66 & $\underline{8.96}$ & 8.00 \\
\hline NIG model & 6.25 & 9.09 & 9.91 & 8.60 & 5.46 & 8.72 & 9.63 & 8.03 \\
\hline VG model & 6.66 & 9.37 & 10.09 & 8.88 & 5.83 & 9.07 & 9.77 & 8.35 \\
\hline$t$ model & $\underline{6.03}$ & $\underline{8.69}$ & 9.49 & 8.24 & $\underline{5.30}$ & $\underline{8.36}$ & 9.20 & 7.71 \\
\hline GH model & 6.13 & 8.70 & 9.54 & $\overline{8.27}$ & 5.35 & 8.37 & 9.25 & $\overline{7.73}$ \\
\hline \multicolumn{9}{|l|}{ All } \\
\hline Black-Scholes & 26.60 & 15.26 & 11.38 & 17.48 & 20.99 & 13.30 & 9.56 & 13.98 \\
\hline FMLS model & 21.17 & 10.49 & 10.86 & 13.47 & 15.55 & 8.60 & 8.55 & 10.02 \\
\hline NIG model & 13.40 & 9.18 & 9.54 & 10.27 & 8.94 & 7.22 & 7.35 & 7.61 \\
\hline VG model & 13.19 & 9.35 & 9.89 & 10.38 & 9.56 & 7.37 & 7.63 & 7.89 \\
\hline$t$ model & $\underline{9.39}$ & 8.79 & 9.47 & $\underline{9.08}$ & 7.46 & $\underline{6.88}$ & 7.21 & 7.08 \\
\hline GH model & 9.40 & 8.78 & 9.47 & $\underline{9.08}$ & 7.46 & $\underline{6.88}$ & 7.21 & $\overline{7.08}$ \\
\hline
\end{tabular}

The smallest error measure within each group is italicised and underlined. The bold values show the overall error measure. Parameters are estimated using all options on a given day regardless of their time-to-maturity and moneyness (an average of 60 observations per day, and 250 days in year 2012). Pricing errors are classified by time-to-maturity and moneyness in accordance with the categories in Table ??. The errors are then averaged across the 250 testing days collectively. The sample size is $n=15,058$. 


\subsection{Orthogonality Test}

A comprehensive cross-sectional analysis of the pricing errors leads us to the second yardstick for assessing model adequacy. A well-specified model should not only minimise some loss function on pricing errors (e.g., RMSPE and MAPE) but also yield individual pricing errors that are independent of moneyness, time-to-maturity and interest rates (?). Furthermore, the pricing error measure used for assessing model adequacy needs to accord with that used for parameter estimation (?). To ensure consistency with the model estimation procedure in Section ??, we therefore evaluate the model fitting using the sequence of option pricing errors relative to the observed option prices:

$$
\epsilon_{i t}=\frac{O_{i t}\left(\hat{\Theta}_{t}\right)-O_{i t}}{O_{i t}}
$$

where $O_{i t}$ is the observed option price in dollars, and $O_{i t}\left(\hat{\Theta}_{t}\right)$ is the option price estimated by the model. We investigate the orthogonality of the relative pricing errors by regressing $\epsilon_{i t}$ on some option and market attributes, including moneyness (defined as the ratio of the strike to the spot price), squared moneyness, time-to-maturity, squared time-to-maturity, and the risk-free interest rate. More specifically, we consider the following regression model for the orthogonality test:

$$
\epsilon_{i t}=b_{0}+b_{1}\left(\frac{K_{i}}{S_{t}}\right)+b_{2}\left(\frac{K_{i}}{S_{t}}\right)^{2}+b_{3} \tau_{i t}+b_{4} \tau_{i t}^{2}+b_{5} r_{t}+e_{i t}
$$

where $K_{i}$ and $\tau_{i t}$ are the strike price and time-to-maturity of the $i^{t h}$ option at time $t, S_{t}$ is the S\&P 500 Index level at time $t, r_{t}$ is the risk-free interest rate at time $t$, and $e_{i t}$ is the random error term. If the model is well specified, we expect close-to-zero regression coefficients and a low regression $R^{2}$, both signifying greater orthogonality of the relative pricing errors.

We run the regression on the entire 250-day sample consisting of 15,058 observations of relative pricing error. The results are presented in Table ??. As revealed by the $t$ tests on the regression coefficients and the $F$ tests on the regression $R^{2}$, all models are generally misspecified to various degrees. Comparing the extent of error orthogonality across models, the Black-Scholes model yields the highest regression $R^{2}(72.0 \%)$, followed by the FMLS model (46.5\%), the GH model (34.6\%), the $t$ model (34.5\%), the VG model $(32.8 \%)$, and the NIG model $(28.7 \%)$. While NIG model achieves the least predictable relative pricing errors, its total sum of squared pricing errors (TSS) is larger in magnitude compared to the $t$ and GH models, which have the smallest TSS among all models being considered. There is a trade-off to be made - from a practitioner's perspective the overall pricing accuracy is likely to take precedence over the orthogonality of pricing errors when choosing among the models. 
Table 5: Results of orthogonality test.

\begin{tabular}{|c|c|c|c|c|c|c|}
\hline Explanatory variable & Black-Scholes & FMLS & NIG & VG & $t$ & GH \\
\hline Intercept & $\begin{array}{l}-103.14 \\
(0.943)^{*}\end{array}$ & $\begin{array}{l}-54.58 \\
(0.943)^{*}\end{array}$ & $\begin{array}{l}-13.11 \\
(0.856)^{*}\end{array}$ & $\begin{array}{l}-18.96 \\
(0.827)^{*}\end{array}$ & $\begin{array}{l}-10.78 \\
(0.742)^{*}\end{array}$ & $\begin{array}{l}-10.92 \\
(0.742)^{*}\end{array}$ \\
\hline Moneyness & $\begin{array}{l}204.36 \\
(1.887)^{*}\end{array}$ & $\begin{array}{l}107.88 \\
(1.880)^{*}\end{array}$ & $\begin{array}{l}24.95 \\
(1.709)^{*}\end{array}$ & $\begin{array}{l}36.72 \\
(1.654)^{*}\end{array}$ & $\begin{array}{l}20.37 \\
(1.483)^{*}\end{array}$ & $\begin{array}{l}20.63 \\
(1.483)^{*}\end{array}$ \\
\hline Moneyness $^{2}$ & $\begin{array}{l}-101.07 \\
(0.944)^{*}\end{array}$ & $\begin{array}{l}-53.16 \\
(0.937)^{*}\end{array}$ & $\begin{array}{l}-11.75 \\
(0.853)^{*}\end{array}$ & $\begin{array}{l}-17.65 \\
(0.827)^{*}\end{array}$ & $\begin{array}{l}-9.48 \\
(0.741)^{*}\end{array}$ & $\begin{array}{l}-9.61 \\
(0.740)^{*}\end{array}$ \\
\hline Time-to-maturity & $\begin{array}{l}0.322 \\
(0.047)^{*}\end{array}$ & $\begin{array}{l}-0.329 \\
(0.049)^{*}\end{array}$ & $\begin{array}{l}-0.550 \\
(0.038)^{*}\end{array}$ & $\begin{array}{l}-0.609 \\
(0.036)^{*}\end{array}$ & $\begin{array}{l}-0.632 \\
(0.027)^{*}\end{array}$ & $\begin{array}{l}-0.630 \\
(0.027)^{*}\end{array}$ \\
\hline Time-to-maturity ${ }^{2}$ & $\begin{array}{l}-1.204 \\
(0.103)^{*}\end{array}$ & $\begin{array}{l}-0.020 \\
(0.107)\end{array}$ & $\begin{array}{l}0.893 \\
(0.085)^{*}\end{array}$ & $\begin{array}{l}0.940 \\
(0.081)^{*}\end{array}$ & $\begin{array}{l}1.070 \\
(0.064)^{*}\end{array}$ & $\begin{array}{l}1.062 \\
(0.064)^{*}\end{array}$ \\
\hline Interest rate & $\begin{array}{l}5.851 \\
(2.249)^{*}\end{array}$ & $\begin{array}{l}6.953 \\
(2.415)^{*}\end{array}$ & $\begin{array}{l}5.493 \\
(2.157)\end{array}$ & $\begin{array}{l}5.653 \\
(2.136)^{*}\end{array}$ & $\begin{array}{l}5.354 \\
(1.831)^{*}\end{array}$ & $\begin{array}{l}4.802 \\
(1.826)^{*}\end{array}$ \\
\hline TSS & 438.39 & 268.73 & 157.32 & 160.16 & 122.63 & 122.62 \\
\hline $\begin{array}{l}R^{2} \\
F \text {-statistic }\end{array}$ & $\begin{array}{l}72.0 \% \\
(7735.1)^{*}\end{array}$ & $\begin{array}{l}46.5 \% \\
(2615.1)^{*}\end{array}$ & $\begin{array}{l}28.7 \% \\
(1211.9)^{*}\end{array}$ & $\begin{array}{l}32.8 \% \\
(1466.7)^{*}\end{array}$ & $\begin{array}{l}34.5 \% \\
(1586.2)^{*}\end{array}$ & $\begin{array}{l}34.6 \% \\
(1595.3)^{*}\end{array}$ \\
\hline
\end{tabular}

S\&P 500 Index put options from 2012 are used, with sample size $n=15,058$. Moneyness is the strike-tospot price ratio, and time-to-maturity is in years. Heteroskedastic standard errors are shown in parentheses, * indicates statistical significance at the $1 \%$ level of significance. The critical $t$-statistics are respectively $t_{(0.005,15052)}= \pm 2.58$ and $t_{(0.025,15052)}= \pm 1.96$. TSS is the total sum of squared errors. At the $1 \%$ level of significance, the critical $F_{(0.01,5,15052)}$-statistic is 3.02 .

\subsection{Out-of-Sample Pricing Performance}

Finally, we examine the out-of-sample pricing performance of the option pricing models. This is motivated from both a practical (price prediction) as well as a statistical consideration. Whilst in-sample pricing performance benefits from having additional parameters, this is usually not the case when it comes to out-of-sample prediction, as prediction accuracy can be penalised by overfitting a model (?).

In order to compute the out-of-sample pricing errors, we first estimate the model over a rolling 5-day training period. Using the calibrated model, we then compute the one-day ahead forecast of the option prices. With the 2012 S\&P 500 Index put options data, there are 245 testing samples and a total of 14,794 pricing errors. Each training sample has on average 301 data points. The out-of-sample performance results are presented in Table ??.

First, we focus on the out-of-sample performance of models in predicting the entire cross-section. The GH model emerges as the sole superior model overall in terms of both RMSPE (15.38\%) and MAPE (11.04\%). Unlike in the in-sample context, the GH model achieves greater out-of-sample accuracy than the $t$ model, which attains an RMSPE of $16.33 \%$ (almost a full percentage point higher than the GH model) and an MAPE of $11.16 \%(0.12$ percentage points higher than the GH model). Among the special cases of the GH model being considered (NIG, VG and $t$ ), the $t$ model is superior. The VG model obtains a $16.73 \%$ RMSPE, and the NIG model obtains a $16.71 \%$ RMSPE. It is evident that the GH model family dominates the the FMLS model $(18.98 \%$ RMSPE, $13.48 \%$ MAPE) and the Black-Scholes model (19.94\% RMSPE) in terms of overall out-of-sample performance.

Next, we compare the out-of-sample performance of the models in different parts of the cross-section. The GH model is the best model for OTM, ATM, short-term and long-term option categories. In particular, it outperforms the next best model by a wide margin for short-term options (by 2.97 percentage points) and for OTM options (by 1.45 percentage points). As in the in-sample setting, the $t$ model fits the ITM options better than the GH model. In all option categories, the NIG model is strictly dominated by the GH model. This highlights the importance of freeing up the index parameter $p$ for optimal out-of-sample performance. We note that some pricing improvements are observed in the out-of-sample context compared to the in-sample context for ITM options (for 
example, ITM option prices as fit by the VG model). This can be explained by the relatively small representation of ITM options (16\%) in the data compared to ATM (41\%) and OTM (43\%) options (see Table ??), such that the ITM options have a relatively weak influence over the in-sample fitting. For the medium-term option prices, the VG, $t$ and GH models perform similarly well. The FMLS model does not exhibit any advantage over the GH model family in forecasting the option prices in the given sample. It performs especially poorly in predicting the prices of short-term, OTM puts (43.01\% RMSPE). This suggests that the heavy tail assumption under the FMLS model may have introduced unnecessary noise that hinders out-of-sample performance, at least during the sample period being considered. Interestingly, the Black-Scholes model provides the best out-of-sample fit for long-term, OTM puts (14.34\% RMSPE), implying that the additional complexity beyond normality as offered by the FMLS and GH models does not facilitate price prediction for this class of put options. 
Table 6: Out-of-sample pricing root mean squared percentage errors (RMSPE) and mean absolute percentage errors (MAPE) in the main estimation.

\begin{tabular}{|c|c|c|c|c|c|c|c|c|}
\hline & \multicolumn{4}{|c|}{ Out-of-sample RMSPE (\%) } & \multicolumn{4}{|c|}{ Out-of-sample MAPE (\%) } \\
\hline & \multicolumn{4}{|c|}{ Time-to-maturity } & \multicolumn{4}{|c|}{ Time-to-maturity } \\
\hline & Short & Medium & Long & All & Short & Medium & Long & All \\
\hline \multicolumn{9}{|c|}{ Out-of-the-money } \\
\hline Black-Scholes & 36.35 & 19.36 & 14.34 & 23.35 & 26.95 & 15.38 & $\underline{11.58}$ & 17.04 \\
\hline FMLS model & 43.01 & 19.20 & $\overline{15.81}$ & 24.93 & 33.59 & 15.53 & 13.03 & 18.35 \\
\hline NIG model & 37.13 & 17.60 & 15.85 & 22.35 & 26.14 & 14.20 & 13.05 & 16.19 \\
\hline VG model & 33.77 & 17.48 & 15.74 & 21.87 & 22.12 & 13.99 & 13.13 & 15.60 \\
\hline$t$ model & 33.63 & 17.27 & 15.35 & 21.66 & 20.68 & $\underline{13.86}$ & 12.66 & 15.09 \\
\hline GH model & 29.31 & $\overline{17.28}$ & 15.32 & 20.21 & $\underline{19.52}$ & 13.89 & 12.65 & 14.85 \\
\hline \multicolumn{9}{|c|}{ At-the-money } \\
\hline Black-Scholes & 27.98 & 17.61 & 10.06 & 18.61 & 23.11 & 15.38 & 8.49 & 15.06 \\
\hline FMLS model & 21.66 & 12.81 & 8.49 & 14.10 & 16.04 & 10.46 & 6.90 & 10.60 \\
\hline NIG model & 16.29 & 10.64 & 8.41 & 11.46 & 12.05 & 8.50 & 6.53 & 8.67 \\
\hline VG model & 20.44 & 9.76 & 7.28 & 12.10 & 14.52 & 7.87 & 5.73 & 8.59 \\
\hline$t$ model & 16.51 & $\overline{10.23}$ & $\overline{7.64}$ & 11.17 & 11.77 & $\overline{8.27}$ & $\overline{6.11}$ & 8.37 \\
\hline GH model & $\underline{15.18}$ & 10.21 & 7.50 & 10.77 & $\underline{11.56}$ & 8.26 & 6.03 & $\underline{8.31}$ \\
\hline \multicolumn{9}{|c|}{ In-the-money } \\
\hline Black-Scholes & 7.14 & 12.10 & 11.59 & 10.98 & 5.92 & 11.26 & 10.93 & 9.87 \\
\hline FMLS model & 6.36 & 9.64 & 9.63 & 8.94 & 5.49 & 8.79 & 8.77 & 7.96 \\
\hline NIG model & 5.93 & 9.39 & 9.43 & 8.67 & 5.07 & 8.57 & 8.66 & 7.71 \\
\hline VG model & 6.45 & 9.35 & $\underline{9.00}$ & 8.66 & 5.50 & 8.61 & $\underline{8.29}$ & 7.78 \\
\hline$t$ model & 5.64 & 9.08 & $\overline{9.29}$ & $\underline{8.39}$ & 4.79 & 8.27 & $\overline{8.58}$ & 7.45 \\
\hline GH model & $\overline{5.70}$ & $\overline{9.13}$ & 9.28 & $\overline{8.43}$ & $\overline{4.80}$ & $\overline{8.32}$ & 8.57 & $\overline{7.48}$ \\
\hline \multicolumn{9}{|l|}{ All } \\
\hline Black-Scholes & 29.88 & 17.60 & 12.30 & 19.94 & 21.70 & 14.68 & 10.13 & 15.10 \\
\hline FMLS model & 30.71 & 15.47 & 12.39 & 18.98 & 21.05 & 12.34 & 9.83 & 13.48 \\
\hline NIG model & 25.90 & 13.89 & 12.38 & 16.71 & 16.37 & 10.94 & 9.67 & 11.72 \\
\hline VG model & 26.01 & 13.55 & 11.96 & 16.73 & 16.31 & 10.61 & 9.30 & 11.52 \\
\hline$t$ model & 24.86 & 13.54 & 11.85 & 16.33 & 14.53 & 10.65 & 9.29 & 11.16 \\
\hline GH model & $\underline{21.89}$ & $\overline{13.54}$ & 11.79 & 15.38 & $\underline{13.93}$ & 10.67 & 9.25 & 11.04 \\
\hline
\end{tabular}

S\&P 500 Index put options during 2012 are used. The smallest error measure within each group is italicised and underlined. The bold values show the overall error measures. Each training sample spans 5 days (on average containing 301 data points) and each testing sample is one day ahead of the training sample (on average containing 60 data points). There are 245 testing days. Parameters are estimated using all options in the entire cross-section of the sample. Pricing errors are classified by time-to-maturity and moneyness in accordance with the categories in Table ??. The errors are then averaged across the 245 testing days collectively. The sample size is $n=14,794$.

\subsection{Robustness Analysis}

The above estimation results are based on the dataset from 2012 in which the US stock market was relatively stable. Indeed, the VIX remains below 30 during the sample period. In this section, we want to investigate how the results will change under a more turbulent market condition. The robustness analysis will be based on a sample consisting of daily put option prices from $4^{\text {th }}$ August 2011 to $13^{\text {th }}$ October 2011 . The period, which spans 50 trading days, was selected according to the criterion that the VIX Index exceeded and remained above the level 30 over the entire duration. The peak of VIX occurred at 48.00 on $8^{\text {th }}$ August 2011, dubbed the "Black 
Monday" in the US stock market as it reacted sharply to the Standard and Poor credit agency's decision to downgrade the credit rating of the US sovereign debt. The sample period is shown in Figure ??.

In the robustness analysis, we revisit the out-of-sample model evaluation based on the new dataset. The sampling and estimation strategy are identical as before (using a 5-day rolling window and the cross-section as training samples to estimate the model and predict the next-day put option prices). The robustness estimation results are presented in Table ?? and summarised in Table ??. Given the superior out-of-sample performance of the flexible GH model in Section ?? (see Table ??) and to simplify the comparison, we do not present the out-of-sample results for the special cases of the GH model in our robustness analysis.

A number of observations are in order. First, the GH model remains the best model in terms of overall out-ofsample performance (15.21\% RMSPE and $11.97 \%$ MAPE). Second, the GH model outperforms the other models in four individual option categories (OTM, short-term, medium-term and long-term). In the ATM and ITM option groups, the FMLS model, which allows for infinite kurtosis in the log-return distribution, surpasses the GH model to be the best predictor. Third, focusing on the nine moneyness and time-to-maturity combinations, the GH model is the best model for OTM put options uniformly over all maturity groups, but the FMLS model performs uniformly the best for ITM puts; the model ranking is somewhat mixed for ATM puts across different maturities.

From these observations, we learn that, among the models under consideration, not a single one of them eclipses the others in predicting every part of the cross-section of put option prices. The flexibility of the GH model contributes to the best overall out-of-sample performance and in particular to the prediction of the OTM put prices. Nevertheless, under turbulent market conditions, the advantage of the heavy-tailed log-return distribution under the FMLS model is manifested when predicting the ITM put prices, which are sensitive to the extreme left tail of the spot price distribution.

We note that all models perform noticeably more poorly during the turbulent period in terms of out-of-sample RMSPE. Furthermore, the differential in the predictive ability of the more complex models relative to BlackScholes becomes narrower (e.g, during the normal sample period in the main analysis, the GH and FMLS models outperform the BS model overall by 4.56 and 0.96 percentage points, respectively, in out-of-sample RMSPE; the gaps shrink to 0.93 and 0.56 percentage points, respectively, during the turbulent period). The weaker pricing performance may be attributable to the stringent i.i.d. assumption, which is likely violated during turbulent market conditions. The heightened persistence of high volatilities as perceived by investors in turbulent times is associated with a higher option price; however, this is not directly modelled by the models we considered thus far. This motivates us to consider some of the possible stochastic volatility extensions to the GH option pricing model, to be discussed in the following section. 
Figure 4: The sample periods for main estimation and robustness estimation.

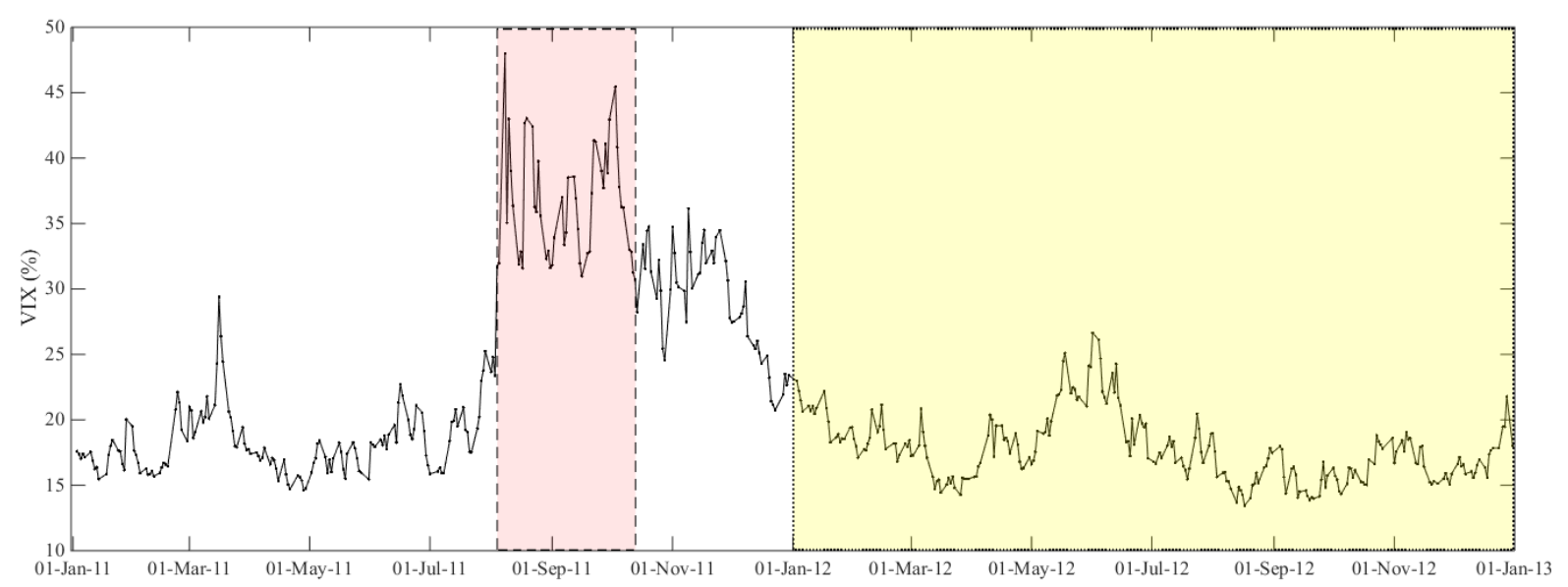

The period used for main estimation spans over 2012 and is shaded in yellow. The period used for robustness estimation spans from August 4 to October 13 (50 trading days) and is shaded in red. The period used for robustness estimation was chosen such that the daily VIX (plotted) stays above 30 continuously over the period.

Table 7: Out-of-sample pricing root mean squared percentage errors (RMSPE) and mean absolute percentage errors (MAPE) in the robustness estimation.

\begin{tabular}{|c|c|c|c|c|c|c|c|c|}
\hline & \multicolumn{4}{|c|}{ Out-of-sample RMSPE (\%) } & \multicolumn{4}{|c|}{ Out-of-sample MAPE (\%) } \\
\hline & \multicolumn{4}{|c|}{ Time-to-maturity } & \multicolumn{4}{|c|}{ Time-to-maturity } \\
\hline & Short & Medium & Long & All & Short & Medium & Long & All \\
\hline \multicolumn{9}{|c|}{ Out-of-the-money } \\
\hline Black-Scholes & 27.04 & 18.52 & 15.94 & 19.94 & 21.63 & 14.80 & 12.31 & 15.51 \\
\hline FMLS model & 26.08 & 17.98 & 15.84 & 19.39 & 19.97 & 14.49 & 12.27 & 15.00 \\
\hline GH model & 24.46 & 17.13 & 15.26 & 18.41 & 19.10 & 13.88 & 12.12 & 14.46 \\
\hline \multicolumn{9}{|c|}{ At-the-money } \\
\hline Black-Scholes & 18.00 & $\underline{13.11}$ & 14.61 & 14.59 & 14.67 & 10.72 & 11.76 & 11.77 \\
\hline FMLS model & 15.79 & 13.42 & 14.29 & 14.16 & 12.60 & $\overline{10.98}$ & 11.47 & 11.43 \\
\hline GH model & 14.68 & 13.96 & $\overline{14.43}$ & $\overline{14.24}$ & 11.67 & 11.55 & $\overline{11.71}$ & $\overline{11.62}$ \\
\hline \multicolumn{9}{|l|}{ In-the-money } \\
\hline Black-Scholes & 9.83 & 11.18 & 15.35 & 11.92 & 8.28 & 9.24 & 12.47 & 9.71 \\
\hline FMLS model & 8.12 & 10.41 & 14.79 & $\underline{11.05}$ & 7.08 & 8.42 & 11.84 & 8.84 \\
\hline GH model & $\overline{8.46}$ & $\overline{10.52}$ & $\overline{14.94}$ & $\overline{11.21}$ & $\overline{7.36}$ & $\overline{8.57}$ & $\overline{12.16}$ & $\overrightarrow{9.06}$ \\
\hline \multicolumn{9}{|l|}{ All } \\
\hline Black-Scholes & 20.03 & 14.84 & 15.25 & 16.14 & 15.24 & 11.78 & 12.10 & 12.57 \\
\hline FMLS model & 18.65 & 14.55 & 14.97 & 15.58 & 13.57 & 11.53 & $\underline{11.83}$ & 12.03 \\
\hline GH model & 17.58 & 14.39 & 14.84 & $\underline{15.21}$ & $\underline{13.03}$ & 11.57 & 11.95 & 11.97 \\
\hline
\end{tabular}

S\&P 500 Index put options during 2012 are used. The smallest error measure within each group is italicised and underlined. The bold values show the overall error measures. Each training sample is 5 days (on average containing 289 data points) and each testing sample is one day ahead of the training sample (on average containing 58 data points). There are 45 testing days. The sample size is $n=2,598$. 
Table 8: A summary of the superior model(s) based on RMSPE by option categories

\begin{tabular}{lccc}
\hline & In-sample & Out-of-sample (Main) & Out-of-sample (Robustness) \\
\hline Out-of-the-money & $\mathrm{GH}, t$ & $\mathrm{GH}$ & $\mathrm{GH}$ \\
At-the-money & $\mathrm{GH}$ & $\mathrm{GH}$ & FMLS \\
In-the-money & $t$ & $\mathrm{GH}, t$ & FMLS \\
Short-term & $t$ & $\mathrm{GH}$ & $\mathrm{GH}$ \\
Medium-term & $\mathrm{GH}$ & $\mathrm{GH}, t$ & $\mathrm{GH}$ \\
Long-term & $\mathrm{GH}, t$ & $\mathrm{GH}$ & $\mathrm{GH}$ \\
All & $\mathrm{GH}, t$ & $\mathrm{GH}$ & $\mathrm{GH}$ \\
\hline
\end{tabular}

A summary of the superior model(s) based on RMSPE in each of the option categories for the in-sample estimation and two out-of-sample predictions as reported in Tables 4, 6 and 7. For the out-of-sample (robustness) analysis, the comparison is done between only the BSM, FMLS and GH models, while for the in-sample and out-of-sample (main) analyses, the GH sub-models are also compared. See Table ?? for the definition of the option categories for moneyness and time-to-maturity.

\section{Stochastic Volatility Extensions}

So far, we have formulated and empirically tested the GH model by assuming stationary and independent increments in the spot price dynamics. In the literature, however, it was documented that the volatility of log-returns displays strong persistence and stochastic behaviors to the extent that the i.i.d. assumption is rejected empirically. This suggests that a GH model that could account for serial dependence in volatility is likely to reduce the pricing errors as observed in the previous empirical study.

Building on the time-changed Lévy process modelling framework in Section 11, it is possible to introduce stochastic volatility in a similar spirit of Carr, Geman, Madan and Yor (CGMY, 2003), ?, and ?. We start with the baseline GH model, in which the source of randomness is the subordinated Brownian motion $B(g(t))$, where $g(t)$ is the GIG process. Next, noting that $B(g(t))$ is a Lévy process with stationary and independent increments, we may construct a time-changed Lévy process as in (1) by setting $L(t)=B(g(t))$ and define a time deformation process $T(t)$ that satisfies the three conditions in Section 1 The resultant time-changed Lévy process $Z(t)=L(T(t))$ - a subordinated GH process - is then treated as the random source that drives the log-return process in $(3)$.

To induce stochastic volatilities, the process $T(t)$ should exhibit time-dependent dynamics. One way to achieve this is to set $T(t)$ to be the integral of a mean-reverting process. More precisely, it is given by

$$
\begin{aligned}
T(t) & =\int_{0}^{t} h(u) d u, \\
d h(t) & =\kappa[1-h(t)] d t+\lambda \sqrt{h(t)} d \tilde{W}(t) .
\end{aligned}
$$

The process $h(t)$ represents the activity rate process which determines the speed at which the business clock runs. It is modelled by the square-root mean-reverting process that solves the stochastic differential equation (??), where $\kappa$ controls the rate of mean reversion, $\lambda$ represents the volatility of the activity rate process, and $\tilde{W}(t)$ is a standard Wiener process possibly correlated with the standard Wiener process $W(t)$ in (??) ${ }^{10}$ The long-run activity rate is set to one so that the normalising restriction on $T(t)$ is satisfied. The characteristic function of $T(t)$ is given by (?)

$$
\phi_{T_{t}}(u)=\exp \left(\frac{\kappa^{2} t}{\lambda^{2}}\right)+\frac{2 i u h(0)}{\kappa+\psi \operatorname{coth}(\psi t / 2)}\left[\cosh \left(\frac{\psi t}{2}\right)+\frac{\kappa}{\psi} \sinh \left(\frac{\psi t}{2}\right)\right]^{-\frac{2 \kappa}{\lambda^{2}}},
$$

where $\psi=\sqrt{\kappa^{2}-2 \lambda^{2} i u}$. This allows us to obtain the characteristic function of the log-price using 22, and 44.

\footnotetext{
${ }^{10}$ We may set $\tilde{W}(t)=\rho W(t)+\sqrt{1-\rho^{2}} \breve{W}(t)$, where $W(t)$ and $\breve{W}(t)$ are independent standard Wiener processes.
} 
The European option prices under the stochastic volatility flexible GH model can then be calculated using (??) and (??).

There is an alternative modelling strategy that allows for long-range dependence in stochastic volatility within the subordinated Brownian motion framework introduced in Section $1^{11}$ Under the GH option pricing model of ?, serial dependence of stock returns is modelled by a background-driving Lévy process (BDLP) (?) construction on the subordinator. More specifically, the subordinator $g(t)$ is defined as a superposition of OU processes, each of which is driven by an independent BDLP and has a GIG marginal distribution. The important feature is that the increments of $g(t)$ form an autocorrelated sequence whose marginal distribution remains to be GIG by selfdecomposability (?). The autocorrelation in the increments of $g(t)$ may induce short- or long-range dependence in squared stock returns. Compared our extended GH model to Finlay and Seneta's model, their European option pricing formula involves two-dimensional integrations. We also note that even in the i.i.d. case, the two models have different martingale adjustments and different parametrisations under the risk-neutral measure 12 One future direction of research is to reconcile the models and investigate their empirical performance in the presence of stochastic volatility.

\section{Conclusion}

Insight into the risk-neutral distribution of logarithmic stock returns is vital to the fitting and prediction of option prices. In this paper, we develop a flexible, four-parameter GH option pricing model in the time-changed Lévy process framework, and present six three-parameter special cases: the VG, $t$, hyperbolic, reciprocal hyperbolic, normal inverse Gaussian, and normal reciprocal inverse Gaussian option pricing models. In respect of the seven models' properties, the flexible GH model and its special cases generalise the Black-Scholes model by allowing the passage of economic time to be stochastic. As such, the GH model family forms a class of time-subordinated models that can cope with yet another facet of the unpredictable financial market. In addition, the subordination to a drifted Brownian motion entails that the class of GH processes are able to capture excess kurtosis and skewness. Using the characteristic function approach of ?, we obtain an explicit pricing formula for European options under the baseline GH model. The option pricing formula takes a convenient semi-closed form. With judicious choices of parametrisation and variable transformation, all the four model parameters can be estimated freely. These features greatly facilitate the empirical study on the GH option pricing model.

Using S\&P 500 Index options, we conduct an extensive empirical study on the GH option pricing model and some of its special cases, and compare the empirical performance against some benchmark option pricing models that assume i.i.d. spot returns, including the Black-Scholes model and the finite moment log-stable (FMLS) model (?). Our empirical analysis suggests that the GH model generally delivers the best in-sample fit and outof-sample prediction for all put option prices in the cross section. The same conclusion holds for the robustness analysis in which the sample spans a more turbulent period, although the heavy-tail FMLS option pricing model yields more accurate out-of-sample forecast for some specific option categories. Among the NIG, VG and $t$ special cases, the $t$ model seems to be the most tenable model for pricing options. Remarkably, the $t$ model's average in-sample fit is better than that of the VG model (?) for all option types - a result which is corroborated by the GH model's parameter estimates.

In this paper, we concentrate on the empirical study of the baseline GH model, which belongs to class of the Lévy-based option pricing models that assumes independent and stationary increments in the underlying spot price process. However, the i.i.d. assumption on the spot returns can be crippling in view of the bulk of well-known stylised facts regarding financial asset returns. Leveraging the proliferous time-changed Lévy process framework (?, ?, and ?), we present a stochastic volatility extension to the baseline model that employs the mean-reverting OU process for time deformation. Empirical studies on this extension is left for future work.

\footnotetext{
${ }^{11}$ The option pricing models for assets with long range dependence were pioneered by ?, and ?. Special cases of the GH distribution have since been employed in short- and long-range dependence models, such as the $t$ distribution by ?,?, and?, the VG distribution by ?, and ?, and the normal inverse Gaussian distribution by ??.

${ }^{12}$ Unlike our model, ? do not impose the normalisation restriction $E[g(1)]=1$ on the subordinator. Instead, their martingale condition implies a restriction on the skewness parameter $\theta=-\frac{1}{2} \sigma^{2}$, while $\theta$ is a free parameter under our model.
} 


\section{Appendix A}

\section{The characteristic function of the logarithmic stock price}

First, we derive the value of the martingale correction factor, denoted by $\omega_{t}$, as $-\log \left(\phi_{Z_{t}}(-i)\right)$. Assuming no arbitrage and by risk-neutral pricing, the discounted stock price process $\left\{S_{t} e^{-(r-q) t}\right\}$ follows a martingale under the risk-neutral probability measure $Q$. This means that, for any positive $t$,

$$
S_{0}=\mathrm{E}^{Q}\left[S_{t} e^{-(r-q) t} \mid F_{0}\right]
$$

where $F_{0}$ is the information set at time 0 , and $\mathrm{E}^{Q}\left[\cdot \mid F_{0}\right]$ represents the conditional expectation taken with respect to $Q$.

Given the log stock price model in (3), the martingale condition is satisfied by applying a martingale correction factor $\omega_{t}$ to the drift of the log-return process, as follows:

$$
S_{0}=\mathrm{E}^{Q}\left[S_{0} \exp \left\{(r-q) t+\omega_{t}+Z_{t}-(r-q) t\right\} \mid F_{0}\right]
$$

Solving for $\omega_{t}$, we obtain:

$$
\begin{aligned}
1 & =\mathrm{E}^{Q}\left[\exp \left\{\omega_{t}+Z_{t}\right\} \mid F_{0}\right] \\
1 & =e^{\omega_{t}} \mathrm{E}^{Q}\left[e^{Z_{t}} \mid F_{0}\right] \\
e^{-\omega_{t}} & =\mathrm{E}^{Q}\left[e^{Z_{t}} \mid F_{0}\right] \\
\omega_{t} & =-\log \left(\mathrm{E}^{Q}\left[e^{Z_{t}} \mid F_{0}\right]\right) \\
\omega_{t} & =-\log \left(\phi_{Z_{t}}(-i)\right)
\end{aligned}
$$

This martingale correction factor is the same as the one in CGMY (2003, equation (4.7), p.358) using ordinary exponential transform.

We then obtain (4), by expressing the characteristic function of the log spot price $\phi_{\log S_{t}}(u)$ in terms of $\phi_{Z_{t}}(u)$, the characteristic function of $Z(t)$, as follows:

$$
\begin{aligned}
\phi_{\log S_{t}}(u) & =\mathrm{E}^{Q}\left[\exp \left\{i u \log \left(S_{t}\right)\right\} \mid F_{0}\right] \\
& =\mathrm{E}^{Q}\left[S_{t}^{i u} \mid F_{0}\right] \\
& =\mathrm{E}^{Q}\left[S_{0}^{i u} \exp \left\{i u\left[(r-q) t-\log \left(\phi_{Z_{t}}(-i)\right)+Z_{t}\right]\right\}\right] \\
& =S_{0}^{i u} \exp \left\{i u(r-q) t-\log \left(\phi_{Z_{t}}(-i)\right)\right\} \phi_{Z_{t}}(u)
\end{aligned}
$$

\section{Appendix B}

\section{Derivations for the Flexible GH, VG and $t$ Option Pricing Models}

\section{B.1 The Flexible GH Model}

The characteristic function of the GIG distribution is given by

$$
\begin{aligned}
\phi_{g_{1}}(u) & =\left(\frac{\gamma^{2}}{\gamma^{2}-2 i u}\right)^{\frac{p}{2}} \frac{K_{p}\left(\sqrt{\delta^{2}\left(\gamma^{2}-2 i u\right)}\right)}{K_{p}(\gamma \delta)} \\
& =\left(1-\frac{2}{\gamma^{2}} i u\right)^{-\frac{p}{2}} \frac{K_{p}\left(\zeta \sqrt{1-\frac{2}{\gamma^{2}} i u}\right)}{K_{p}(\zeta)}
\end{aligned}
$$


where $\zeta=\gamma \delta, \gamma^{2}$ is given by Equation 5 and $K_{j}(\cdot)$ is the modified Bessel function of the third kind with index $j$. Using [2], we obtain

$$
\begin{aligned}
\phi_{Z_{1}}(u) & =\phi_{g_{1}}\left(u \theta+\frac{i \sigma^{2} u^{2}}{2}\right) \\
& =\left(1-\frac{2}{\gamma^{2}}\left(i u \theta-\frac{1}{2} \sigma^{2} u^{2}\right)\right)^{-\frac{p}{2}} \frac{K_{p}\left(\zeta \sqrt{1-\frac{2}{\gamma^{2}}\left(i u \theta-\frac{1}{2} \sigma^{2} u^{2}\right)}\right)}{K_{p}(\zeta)} .
\end{aligned}
$$

It follows from (4) that

$$
\begin{aligned}
\phi_{\log S_{t}}(u) & =S_{0}^{i u} \exp \{i u(r-q+\omega) t\} \phi_{X_{1}}(u)^{t} \\
& =S_{0}^{i u} \exp \{i u(r-q+\omega) t\}\left[1-\frac{2}{\gamma^{2}}\left(i u \theta-\frac{1}{2} \sigma^{2} u^{2}\right)\right]^{-\frac{p t}{2}} \times \\
& {\left[\frac{K_{p}\left(\zeta \sqrt{1-\frac{2}{\gamma^{2}}\left(i u \theta-\frac{1}{2} \sigma^{2} u^{2}\right)}\right)}{K_{p}(\zeta)}\right]^{t}, }
\end{aligned}
$$

where the unit time drift adjustment can be computed as

$$
\begin{aligned}
\omega & =-\log \phi_{X_{1}}(-i) \\
& =-\frac{p}{2} \log \left[\left(1-\frac{2}{\gamma^{2}}\left(\theta+\frac{1}{2} \sigma^{2}\right)\right)\right]+\log \left[\frac{K_{p}\left(\zeta \sqrt{1-\frac{2}{\gamma^{2}}\left(\theta+\frac{1}{2} \sigma^{2}\right)}\right)}{K_{p}(\zeta)}\right],
\end{aligned}
$$

for $\theta<\left(\frac{\gamma^{2}}{2}-\frac{\sigma^{2}}{2}\right)$.

\section{Distributional Properties of $Z_{1}$ under the GH Model}

Let us start by studying the statistical properties of $Z_{1}$. First, we derive the probability density function of the generalised hyperbolic distribution, which is the distribution of the random variable $Z_{1}$ in the log-price specification (3) under the flexible GH option pricing model.

Let $\mathrm{N}\left(x ; \mu, \sigma^{2}\right)$ denote the density of a normal distribution with mean $\mu$ and variance $\sigma^{2}$. Suppose, conditional on $g_{1}$, that $X_{1}$ follows a normal distribution with mean $\theta g_{1}$ and variance $\sigma^{2} g_{1}$, and that $g_{1}$ follows a GIG with probability density function $f_{g_{1}}(g)$, given by

$$
\operatorname{GIG}(p, \delta, \gamma): \quad f_{g_{1}}(g)=\frac{\left(\frac{\gamma}{\delta}\right)^{p}}{2 K_{p}(\gamma \delta)} g^{p-1} \exp \left\{-\frac{1}{2}\left(\gamma^{2} g+\frac{\delta^{2}}{g}\right)\right\}
$$

where $K_{j}(\cdot)$ is the modified Bessel function of the third kind with index $j$. Then $Z_{1}$ will follow a GH distribution with its density function $f_{Z_{1}}(x)$ given by, for any real value $x$,

$$
\begin{aligned}
f_{Z_{1}}(x) & =\int_{0}^{\infty} \mathrm{N}\left(x ; \theta g, \sigma^{2} g\right) f_{g_{1}}(g) d g \\
& =\int_{0}^{\infty} \frac{1}{\sqrt{2 \pi \sigma^{2} g}} \exp \left\{-\frac{(x-\theta g)^{2}}{2 \sigma^{2} g}\right\} \cdot \frac{\left(\frac{\gamma}{\delta}\right)^{p}}{2 K_{p}(\gamma \delta)} g^{p-1} \exp \left\{-\frac{1}{2}\left(\gamma^{2} g+\frac{\delta^{2}}{g}\right)\right\} d g \\
& =\frac{\left(\frac{\gamma}{\delta}\right)^{p}}{2 \sqrt{2 \pi \sigma^{2}} K_{p}(\gamma \delta)} \int_{0}^{\infty} g^{p-\frac{3}{2}} \exp \left\{-\frac{1}{2}\left[\frac{(x-\theta g)^{2}}{\sigma^{2} g}+\gamma^{2} g+\frac{\delta^{2}}{g}\right]\right\} d g \\
& =\frac{\left(\frac{\gamma}{\delta}\right)^{p}}{2 \sqrt{2 \pi \sigma^{2}} K_{p}(\gamma \delta)} e^{\frac{\theta}{\sigma^{2}} x} \int_{0}^{\infty} g^{p-\frac{3}{2}} \exp \left\{-\frac{1}{2}\left[\left(\frac{\theta^{2}}{\sigma^{2}}+\gamma^{2}\right) g+\left(\frac{x^{2}}{\sigma^{2}}+\delta^{2}\right) \frac{1}{g}\right]\right\} d g \\
& =\frac{\left(\frac{\gamma}{\delta}\right)^{p}}{\sqrt{2 \pi \sigma^{2}} K_{p}(\gamma \delta)} e^{\frac{\theta}{\sigma^{2}} x}\left(\frac{\frac{x^{2}}{\sigma^{2}}+\delta^{2}}{\frac{\theta^{2}}{\sigma^{2}}+\gamma^{2}}\right)^{\frac{p}{2}-\frac{1}{4}} K_{p-\frac{1}{2}}\left(\sqrt{\left(\frac{\theta^{2}}{\sigma^{2}}+\gamma^{2}\right)\left(\frac{x^{2}}{\sigma^{2}}+\delta^{2}\right)}\right) .
\end{aligned}
$$


The last equality uses the fact that, for all $\eta, v>0$,

$$
\int_{0}^{\infty} x^{j-1} e^{-\frac{1}{2}\left(\eta x+\frac{v}{x}\right)} d x=2\left(\frac{v}{\eta}\right)^{\frac{j}{2}} K_{j}(\sqrt{\eta v}) .
$$

The parameter restriction 5 that $\gamma^{2}=\zeta \frac{K_{p+1}(\zeta)}{K_{p}(\zeta)}$ (where $\zeta=\gamma \delta$ ) coming from the normalisation $\mathrm{E}\left[g_{1}\right]=1$ still holds under the time-changed Lévy process framework.

The first four standardised central moments of $Z_{1}$ under the GH model are given by

$$
\begin{aligned}
E\left(Z_{1}\right) & =\frac{\theta \delta}{\gamma} \frac{K_{p+1}(\delta \gamma)}{K_{p}(\delta \gamma)}, \\
\operatorname{Var}\left(Z_{1}\right) & =\frac{\sigma^{2} \delta}{\gamma} \frac{K_{p+1}(\delta \gamma)}{K_{p}(\delta \gamma)}+\frac{\theta^{2} \delta^{2}}{\gamma^{2}}\left[\frac{K_{p+2}(\delta \gamma)}{K_{p}(\delta \gamma)}-\left(\frac{K_{p+1}(\delta \gamma)}{K_{p}(\delta \gamma)}\right)^{2}\right] \\
\operatorname{skew}\left(Z_{1}\right) & =\frac{E\left[\left(Z_{1}-E\left(Z_{1}\right)\right)^{3}\right]}{\operatorname{Var}\left(Z_{1}\right)^{3 / 2}} \\
& =\operatorname{Var}\left(Z_{1}\right)^{-3 / 2}\left\{\frac{\theta^{3} \delta^{3}}{\gamma^{3}}\left[\frac{K_{p+3}(\delta \gamma)}{K_{p}(\delta \gamma)}-3 \frac{K_{p+2}(\delta \gamma) K_{p+1}(\delta \gamma)}{K_{p}^{2}(\delta \gamma)}+2\left(\frac{K_{p+1}(\delta \gamma)}{K_{p}(\delta \gamma)}\right)^{3}\right]\right. \\
& \left.+3 \frac{\sigma^{2} \theta \delta^{2}}{\gamma^{2}}\left[\frac{K_{p+2}(\delta \gamma)}{K_{p}(\delta \gamma)}-\left(\frac{K_{p+1}(\delta \gamma)}{K_{p}(\delta \gamma)}\right)^{2}\right]\right\}, \\
\operatorname{kur}\left(Z_{1}\right) & =\frac{E\left[\left(Z_{1}-E\left(Z_{1}\right)\right)^{4}\right]}{\operatorname{Var}\left(Z_{1}\right)^{2}} \\
& =\operatorname{Var}\left(Z_{1}\right)^{-2}\left\{\frac{\theta^{4} \delta^{4}}{\gamma^{4}}\left[\frac{K_{p+4}(\delta \gamma)}{K_{p}(\delta \gamma)}-4 \frac{K_{p+3}(\delta \gamma) K_{p+1}(\delta \gamma)}{K_{p}^{2}(\delta \gamma)}+6 \frac{K_{p+2}(\delta \gamma) K_{p+1}^{2}(\delta \gamma)}{K_{p}^{3}(\delta \gamma)}-3\left(\frac{K_{p+1}(\delta \gamma)}{K_{p}(\delta \gamma)}\right)^{4}\right]\right. \\
& \left.+\frac{\sigma^{2} \theta^{2} \delta^{3}}{\gamma^{3}}\left[6 \frac{K_{p+3}(\delta \gamma)}{K_{p}(\delta \gamma)}-12 \frac{K_{p+2}(\delta \gamma) K_{p+1}(\delta \gamma)}{K_{p}^{2}(\delta \gamma)}+6\left(\frac{K_{p+1}(\delta \gamma)}{K_{p}(\delta \gamma)}\right)^{3}\right]+3 \sigma^{4} \frac{\delta^{2}}{\gamma^{2}} \frac{K_{p+2}(\delta \gamma)}{K_{p}(\delta \gamma)}\right\}
\end{aligned}
$$

Applying the normalisation $E\left[g_{1}\right]=1$, and denoting $\zeta=\delta \gamma$, we obtain the parameter restriction $\gamma^{2}=\zeta \frac{K_{p+1}(\zeta)}{K_{p}(\zeta)}$. The four standardised central moments of $Z_{1}$ then become

$$
\begin{aligned}
E\left(Z_{1}\right) & =\theta \\
\operatorname{Var}\left(Z_{1}\right) & =\sigma^{2}+\theta^{2}\left[\frac{K_{p+2}(\zeta) K_{p}(\zeta)}{K_{p+1}^{2}(\zeta)}-1\right], \\
\operatorname{skew}\left(Z_{1}\right) & =\operatorname{Var}\left(Z_{1}\right)^{-3 / 2}\left\{\theta^{3}\left[\frac{K_{p+3}(\zeta) K_{p}^{2}(\zeta)}{K_{p+1}^{3}(\zeta)}-3 \frac{K_{p+2}(\zeta) K_{p}(\zeta)}{K_{p+1}^{2}(\zeta)}+2\right]\right. \\
& \left.+3 \sigma^{2} \theta\left[\frac{K_{p+2}(\zeta) K_{p}(\zeta)}{K_{p+1}^{2}(\zeta)}-1\right]\right\}, \\
\operatorname{kur}\left(Z_{1}\right) & =\operatorname{Var}\left(Z_{1}\right)^{-2}\left\{\theta^{4}\left[\frac{K_{p+4}(\zeta) K_{p}^{3}(\zeta)}{K_{p+1}^{4}(\zeta)}-4 \frac{K_{p+3}(\zeta) K_{p}^{2}(\zeta)}{K_{p+1}^{3}(\zeta)}+6 \frac{K_{p+2}(\zeta) K_{p}(\zeta)}{K_{p+1}^{2}(\zeta)}-3\right]\right. \\
& \left.+\sigma^{2} \theta^{2}\left[6 \frac{K_{p+3}(\zeta) K_{p}^{2}(\zeta)}{K_{p+1}^{3}(\zeta)}-12 \frac{K_{p+2}(\zeta) K_{p}(\zeta)}{K_{p+1}^{2}(\zeta)}+6\right]+3 \sigma^{4} \frac{K_{p+2}(\zeta) K_{p}(\zeta)}{K_{p+1}^{2}(\zeta)}\right\} .
\end{aligned}
$$

Note that in the symmetric case of $\theta=0$, we have $k u r\left(Z_{1}\right)>3$, by the Turán-type inequality $K_{p+2}(y) K_{p}(y)>$ $K_{p+1}^{2}(y)(?)$.

\section{Distributional Properties of $Z_{t}$ under the GH Model}

Next, let us study the statistical properties of $Z_{t}$. From the infinitely divisibility of a general Lévy process $\left\{Z_{t}\right\}$, the characteristic functions of $Z_{t}$ and $Z_{1}$ are related by $\phi_{Z_{t}}(u)=\left[\phi_{Z_{1}}(u)\right]^{t}$. It follows that their cumulant generating functions (i.e., the log-characteristic functions) are related by $\Psi_{Z_{t}}(u)=t \Psi_{Z_{1}}(u)$. By repeated differentiation, the following relations regarding the cumulants of $Z_{t}$ and $Z_{1}$ hold for all positive integers $n$ :

$$
c_{n}\left(Z_{t}\right):=\frac{1}{i^{n}} \frac{\partial^{n}}{\partial u^{n}} \Psi_{Z_{t}}(0)=t \frac{1}{i^{n}} \frac{\partial^{n}}{\partial u^{n}} \Psi_{Z_{1}}(0)=: t c_{n}\left(Z_{1}\right) .
$$


Using the links between the cumulants and the central moments, the first four central moments of $Z_{t}$ and $Z_{1}$ can be related as follows:

$$
\begin{aligned}
E\left(Z_{t}\right) & =c_{1}\left(Z_{t}\right)=t c_{1}\left(Z_{1}\right)=t E\left(Z_{1}\right), \\
\operatorname{Var}\left(Z_{t}\right) & =c_{2}\left(Z_{t}\right)=t c_{2}\left(Z_{1}\right)=t \operatorname{Var}\left(Z_{1}\right), \\
E\left[\left(Z_{t}-E\left(Z_{t}\right)\right)^{3}\right] & =c_{3}\left(Z_{t}\right)=t c_{3}\left(Z_{1}\right)=t E\left[\left(Z_{1}-E\left(Z_{1}\right)\right)^{3}\right], \\
E\left[\left(Z_{t}-E\left(Z_{t}\right)\right)^{4}\right] & =c_{4}\left(Z_{t}\right)+3 c_{2}\left(Z_{t}\right)^{2}=t c_{4}\left(Z_{1}\right)+3\left[t c_{2}\left(Z_{1}\right)\right]^{2} \\
& =t E\left[\left(Z_{1}-E\left(Z_{1}\right)\right)^{4}\right]+3 t(t-1) \operatorname{Var}\left(Z_{1}\right)^{2} .
\end{aligned}
$$

The skewness and kurtosis of $Z_{t}$ are then given by:

$$
\begin{aligned}
\operatorname{skew}\left(Z_{t}\right) & =\frac{E\left[\left(Z_{t}-E\left(Z_{t}\right)\right)^{3}\right]}{\operatorname{Var}\left(Z_{t}\right)^{3 / 2}}=\frac{t E\left[\left(Z_{1}-E\left(Z_{1}\right)\right)^{3}\right]}{\left[t \operatorname{Var}\left(Z_{1}\right)\right]^{3 / 2}}=\frac{1}{\sqrt{t}} \operatorname{skew}\left(Z_{1}\right), \\
\operatorname{kur}\left(Z_{t}\right) & =\frac{E\left[\left(Z_{t}-E\left(Z_{t}\right)\right)^{4}\right]}{\operatorname{Var}\left(Z_{t}\right)^{2}}=\frac{t E\left[\left(Z_{1}-E\left(Z_{1}\right)\right)^{4}\right]+3 t(t-1) \operatorname{Var}\left(Z_{1}\right)^{2}}{\left[\operatorname{Var}\left(Z_{1}\right)\right]^{2}} \\
& =\frac{1}{t} \operatorname{kur}\left(Z_{1}\right)+3\left(1-\frac{1}{t}\right) .
\end{aligned}
$$

We thus see that, for a general Lévy process, its mean and variance increase with $t$, its skewness diminishes with $t$ at the square-root rate, and its kurtosis approaches that of a normal distribution in the limit as $t \rightarrow \infty$. In the symmetric case of $\theta=0$, we see that $\operatorname{kur}\left(Z_{1}\right)>3$, which implies that $k u r\left(Z_{t}\right)>3$ for all finite $t$.

\section{B.2 The VG Model and its Statistical Properties}

\section{Characteristic Function used for Option Pricing}

The characteristic function of the gamma distribution is given by $\phi_{g_{1}}(u)=(1-i u \nu)^{-\frac{1}{\nu}}$ such that

$$
\phi_{X_{1}}(u)=\left[1-\nu\left(i u \theta-\frac{1}{2} \sigma^{2} u^{2}\right)\right]^{-\frac{1}{\nu}} .
$$

It follows then that the VG option price can be computed using

$$
\phi_{\log S_{t}}(u)=S_{0}^{i u} \exp \{i u(r-q+\omega) t\}\left[1-\nu\left(i u \theta-\frac{1}{2} \sigma^{2} u^{2}\right)\right]^{-\frac{t}{\nu}},
$$

with unit time drift adjustment, $\omega=\frac{1}{\nu} \log \left[1-\nu\left(\theta+\frac{1}{2} \sigma^{2}\right)\right]$, for $\theta<\left(\frac{1}{\nu}-\frac{\sigma^{2}}{2}\right)$.

\section{Distributional Properties of $Z_{1}$ under the VG Model}

Let us study the statistical properties of $Z_{1}$. The density function of $Z_{1}$ under the VG model can be obtained from (??) by letting $\delta \rightarrow 0$ and setting $p=\frac{1}{\nu}>0$, so that $\gamma=\sqrt{2 p}=\sqrt{\frac{2}{\nu}}$.

$$
\begin{aligned}
f_{Z_{1}}(x) & =\frac{\left(\frac{\gamma}{\delta}\right)^{\frac{1}{\nu}}}{\sqrt{2 \pi \sigma^{2}} \Gamma\left(\frac{1}{\nu}\right) 2^{\frac{1}{\nu}-1}(\gamma \delta)^{-\frac{1}{\nu}}} e^{\frac{\theta}{\sigma^{2}} x}\left(\frac{\frac{x^{2}}{\sigma^{2}}}{\frac{\theta^{2}}{\sigma^{2}}+\frac{2}{\nu}}\right)^{\frac{1}{2 \nu}-\frac{1}{4}} K_{\frac{1}{\nu}-\frac{1}{2}}\left(\sqrt{\left(\frac{\theta^{2}}{\sigma^{2}}+\frac{2}{\nu}\right) \frac{x^{2}}{\sigma^{2}}}\right) \\
& =\frac{\gamma^{\frac{2}{\nu}}}{\sqrt{2 \pi \sigma^{2}} \Gamma\left(\frac{1}{\nu}\right) 2^{\frac{1}{\nu}-1}} e^{\frac{\theta}{\sigma^{2}} x}\left(\frac{x^{2}}{\theta^{2}+\frac{2 \sigma^{2}}{\nu}}\right)^{\frac{1}{2 \nu}-\frac{1}{4}} K_{\frac{1}{\nu}-\frac{1}{2}}\left(\frac{x}{\sigma^{2}} \sqrt{\theta^{2}+\frac{2 \sigma^{2}}{\nu}}\right) .
\end{aligned}
$$

This recovers the density function in Madan, Carr and Chang (1998, equation (23)).

Imposing the same limits on the parameters, we obtain the first four standardised central moments under the 
VG model for $\nu>0$ :

$$
\begin{aligned}
E\left(Z_{1}\right) & =\theta \\
\operatorname{Var}\left(Z_{1}\right) & =\sigma^{2}+\theta^{2} \nu, \\
\operatorname{skew}\left(Z_{1}\right) & =\frac{E\left[\left(Z_{1}-E\left(Z_{1}\right)\right)^{3}\right]}{\operatorname{Var}\left(Z_{1}\right)^{3 / 2}} \\
& =\operatorname{Var}\left(Z_{1}\right)^{-3 / 2}\left(2 \theta^{3} \nu^{2}+3 \sigma^{2} \theta \nu\right) \\
\operatorname{kur}\left(Z_{1}\right) & =\frac{E\left[\left(Z_{1}-E\left(Z_{1}\right)\right)^{4}\right]}{\operatorname{Var}\left(Z_{1}\right)^{2}} \\
& =3 \operatorname{Var}\left(Z_{1}\right)^{-2}\left[\theta^{4} \nu^{2}(1+2 \nu)+2 \sigma^{2} \theta^{2} \nu(1+2 \nu)+\sigma^{4}(1+\nu)\right] .
\end{aligned}
$$

In the symmetric case of $\theta=0$, the kurtosis is always greater than 3 as $\nu>0$.

\section{B.3 The $t$ Model}

\section{Characteristic Function used for Option Pricing}

The characteristic function of the reciprocal gamma distribution, $\mathrm{R} \Gamma(a, b)$ is given by

$$
\begin{aligned}
\phi_{g_{1}}(u) & =\frac{2(-b i u)^{a / 2}}{\Gamma(a)} K_{a}[\sqrt{-4 b i u}] \\
& =\frac{2\left(\frac{1}{\nu}-1\right)^{\frac{1}{2 \nu}}}{\Gamma\left(\frac{1}{\nu}\right)}(-i u)^{\frac{1}{2 \nu}} K_{\frac{1}{\nu}}\left[\sqrt{-4\left(\frac{1}{\nu}-1\right) i u}\right]
\end{aligned}
$$

where $a=\frac{1}{\nu}$ and $b=\frac{1}{\nu}-1$ for $0<\nu<1 . K_{j}(\cdot)$ is the modified Bessel function of the third kind with index $j$. Using the same procedure as for the GH and VG derivations, the characteristic function of $X_{1}$ is given by

$$
\phi_{X_{1}}(u)=\frac{2\left(\frac{1}{\nu}-1\right)^{\frac{1}{2 \nu}}}{\Gamma\left(\frac{1}{\nu}\right)}\left[-\left(i u \theta-\frac{1}{2} \sigma^{2} u^{2}\right)\right]^{\frac{1}{2 \nu}} K_{\frac{1}{\nu}}\left[\sqrt{-4\left(\frac{1}{\nu}-1\right)\left(i u \theta-\frac{1}{2} \sigma^{2} u^{2}\right)}\right] .
$$

The characteristic function of $\log S_{t}$ is thus given by

$$
\begin{aligned}
& \phi_{\log S_{t}}(u)=S_{0}^{i u} \exp \{i u(r-q+\omega) t\} \times \\
& {\left[\frac{2\left(\frac{1}{\nu}-1\right)^{\frac{1}{2 \nu}}}{\Gamma\left(\frac{1}{\nu}\right)}\left[-\left(i u \theta-\frac{1}{2} \sigma^{2} u^{2}\right)\right]^{\frac{1}{2 \nu}} K_{\frac{1}{\nu}}\left(\sqrt{-4\left(\frac{1}{\nu}-1\right)\left(i u \theta-\frac{1}{2} \sigma^{2} u^{2}\right)}\right)\right]^{t},}
\end{aligned}
$$

and the unit time drift adjustment,

$$
\omega=-\log \left[\frac{2\left(\frac{1}{\nu}-1\right)^{\frac{1}{2 \nu}}}{\Gamma\left(\frac{1}{\nu}\right)}\left[-\left(\theta+\frac{1}{2} \sigma^{2}\right)\right]^{\frac{1}{2 \nu}} K_{\frac{1}{\nu}}\left(\sqrt{-4\left(\frac{1}{\nu}-1\right)\left(\theta+\frac{1}{2} \sigma^{2}\right)}\right)\right]
$$

for $\theta<-\frac{\sigma^{2}}{2}$.

\section{Distributional Properties of $Z_{1}$ under the $t$ Model}

The density function of $Z_{1}$ under the $t$ model can be obtained from (??) by letting $\gamma \rightarrow 0$ and setting $p=-\frac{1}{\nu}<0$, so that $\delta=\sqrt{2(-p-1)}=\sqrt{2\left(\frac{1}{\nu}-1\right)}$.

$$
f_{Z_{1}}(Z)=\frac{\left(\frac{1}{\nu}-1\right)^{\frac{1}{\nu}}}{\sigma \sqrt{2 \pi} \Gamma\left(\frac{1}{\nu}\right)} e^{\frac{\theta}{\sigma^{2}} Z}\left(\frac{\sqrt{Z^{2}+2\left(\frac{1}{\nu}-1\right) \sigma^{2}}}{\theta}\right)^{-\frac{1}{\nu}-\frac{1}{2}} K_{\frac{1}{\nu}+\frac{1}{2}}\left(\frac{\theta}{\sigma^{2}} \sqrt{Z^{2}+2\left(\frac{1}{\nu}-1\right) \sigma^{2}}\right)
$$

By the variable and parameter transformation, $y=Z / \sigma+\mu, \beta=\theta / \sigma, \alpha=\sqrt{\beta^{2}+\gamma^{2}}, \lambda=-1 / \nu$ and $\delta=$ $\sqrt{2\left(\frac{1}{\nu}-1\right)}$, we recover the density function of the GH-skew- $t$ distribution in Aas and Haff (2001) (see their 
equation (3)).

Imposing the same limits on the parameters, we obtain the first four standardised central moments under the $t$ model and the associated domains of $\nu$ in which the moments exist:

$$
\begin{aligned}
E\left(Z_{1}\right) & =\theta \\
\operatorname{Var}\left(Z_{1}\right) & =\sigma^{2}+\theta^{2} \frac{\nu}{1-2 \nu} \text { for } \nu \in\left(0, \frac{1}{2}\right) \\
\operatorname{skew}\left(Z_{1}\right) & =\frac{E\left[\left(Z_{1}-E\left(Z_{1}\right)\right)^{3}\right]}{\operatorname{Var}\left(Z_{1}\right)^{3 / 2}} \\
& =\operatorname{Var}\left(Z_{1}\right)^{-3 / 2}\left[\theta^{3} \frac{4 \nu^{2}}{(1-2 \nu)(1-3 \nu)}+3 \sigma^{2} \theta \frac{\nu}{1-2 \nu}\right] \text { for } \nu \in\left(0, \frac{1}{3}\right) \\
\operatorname{kur}\left(Z_{1}\right) & =\frac{E\left[\left(Z_{1}-E\left(Z_{1}\right)\right)^{4}\right]}{\operatorname{Var}\left(Z_{1}\right)^{2}} \\
& =3 \operatorname{Var}\left(Z_{1}\right)^{-2}\left[\theta^{4} \frac{\nu^{2}(1+5 \nu)}{(1-2 \nu)(1-3 \nu)(1-4 \nu)}\right. \\
& \left.+2 \sigma^{2} \theta^{2} \frac{\nu(1+\nu)}{(1-2 \nu)(1-3 \nu)}+\sigma^{4} \frac{1-\nu}{1-2 \nu}\right] \text { for } \nu \in\left(0, \frac{1}{4}\right)
\end{aligned}
$$

In the symmetric case of $\theta=0$, the kurtosis is always greater than 3 as $\nu>0$.

\section{Appendix $\mathrm{C}$}

\section{The VG and $t$ Limiting Cases of the GH Model}

The following lemma shows how we may obtain the characteristic functions of $Z_{1}$ under the VG and $t$ models as appropriate limits of that of $Z_{1}$ under the GH model.

Lemma 1 (i) Suppose $p=\frac{1}{\nu}>0, \delta \rightarrow 0$ and $\gamma=\sqrt{\frac{2}{\nu}}$. Then, the GH model reduces to the VG model.

(ii) Suppose $p=-\frac{1}{\nu}<-1, \gamma \rightarrow 0$ and $\delta=\sqrt{2\left(\frac{1}{\nu}-1\right)}$. Then, the GH model reduces to the $t$ model.

Proof. Either $\delta \rightarrow 0$ or $\gamma \rightarrow 0$ implies that $\zeta \rightarrow 0$. Making use of the properties of the modified Bessel function of the third kind with index $j, K_{j}(\cdot)$, that

$$
K_{j}(w) \sim \Gamma(|j|) 2^{|j|-1} w^{-|j|} \quad \text { for } w \downarrow 0,
$$

and that $K_{j}(w)=K_{-j}(w)$, the characteristic function of $X_{1}$ under the GH model (Equation (??)) can be further simplified as follows.

Under the assumptions in (i), we have

$$
\begin{aligned}
\phi_{X_{1}}(u) & =\left(1-\nu\left(i u \theta-\frac{1}{2} \sigma^{2} u^{2}\right)\right)^{-\frac{1}{2 \nu}} \lim _{\delta \rightarrow 0} \frac{\Gamma\left(\frac{1}{\nu}\right) 2^{\frac{1}{\nu}-1}\left(\gamma \delta \sqrt{1-\nu\left(i u \theta-\frac{1}{2} \sigma^{2} u^{2}\right)}\right)^{-\frac{1}{\nu}}}{\Gamma\left(\frac{1}{\nu}\right) 2^{\frac{1}{\nu}-1}(\gamma \delta)^{-\frac{1}{\nu}}} \\
& =\left(1-\nu\left(i u \theta-\frac{1}{2} \sigma^{2} u^{2}\right)\right)^{-\frac{1}{2 \nu}}\left(1-\nu\left(i u \theta-\frac{1}{2} \sigma^{2} u^{2}\right)\right)^{-\frac{1}{2 \nu}} \\
& =\left(1-\nu\left(i u \theta-\frac{1}{2} \sigma^{2} u^{2}\right)\right)^{-\frac{1}{\nu}},
\end{aligned}
$$

which is the corresponding characteristic function under the VG model, given in Equation (??). 
Under the assumptions in (ii), we have

$$
\begin{aligned}
\phi_{X_{1}}(u) & =\lim _{\gamma \rightarrow 0}\left(1-\frac{2}{\gamma^{2}}\left(i u \theta-\frac{1}{2} \sigma^{2} u^{2}\right)\right)^{\frac{1}{2 \nu}} \frac{K_{\frac{1}{\nu}}\left(\delta \sqrt{\gamma^{2}-2\left(i u \theta-\frac{1}{2} \sigma^{2} u^{2}\right)}\right)}{K_{\frac{1}{\nu}}(\delta \gamma)} \\
& =\lim _{\gamma \rightarrow 0}\left(1-\frac{2}{\gamma^{2}}\left(i u \theta-\frac{1}{2} \sigma^{2} u^{2}\right)\right)^{\frac{1}{2 \nu}} \frac{K_{\frac{1}{\nu}}\left(\delta \sqrt{\gamma^{2}-2\left(i u \theta-\frac{1}{2} \sigma^{2} u^{2}\right)}\right)}{\Gamma\left(\frac{1}{\nu}\right) 2^{\frac{1}{\nu}-1}(\gamma \delta)^{-\frac{1}{\nu}}} \\
& =\lim _{\gamma \rightarrow 0} \frac{\delta^{\frac{1}{\nu}}}{\Gamma\left(\frac{1}{\nu}\right) 2^{\frac{1}{\nu}-1}}\left(\gamma^{2}-2\left(i u \theta-\frac{1}{2} \sigma^{2} u^{2}\right)\right)^{\frac{1}{2 \nu}} K_{\frac{1}{\nu}}\left(\delta \sqrt{\gamma^{2}-2\left(i u \theta-\frac{1}{2} \sigma^{2} u^{2}\right)}\right) \\
& =\frac{\delta^{\frac{1}{\nu}}}{\Gamma\left(\frac{1}{\nu}\right) 2^{\frac{1}{\nu}-1}}\left(-2\left(i u \theta-\frac{1}{2} \sigma^{2} u^{2}\right)\right)^{\frac{1}{2 \nu}} K_{\frac{1}{\nu}}\left(\delta \sqrt{-2\left(i u \theta-\frac{1}{2} \sigma^{2} u^{2}\right)}\right) \\
& =\frac{2^{\frac{1}{2 \nu}}\left(\frac{1}{\nu}-1\right)^{\frac{1}{2 \nu}}}{\Gamma\left(\frac{1}{\nu}\right) 2^{\frac{1}{\nu}-1}} 2^{\frac{1}{2 \nu}}\left[-\left(i u \theta-\frac{1}{2} \sigma^{2} u^{2}\right)\right]^{\frac{1}{2 \nu}} K_{\frac{1}{\nu}}\left[\sqrt{-4\left(\frac{1}{\nu}-1\right)\left(i u \theta-\frac{1}{2} \sigma^{2} u^{2}\right)}\right] \\
& =\frac{2\left(\frac{1}{\nu}-1\right)^{\frac{1}{2 \nu}}}{\Gamma\left(\frac{1}{\nu}\right)}\left[-\left(i u \theta-\frac{1}{2} \sigma^{2} u^{2}\right)\right]^{\frac{1}{2 \nu}} K_{\frac{1}{\nu}}\left[\sqrt{-4\left(\frac{1}{\nu}-1\right)\left(i u \theta-\frac{1}{2} \sigma^{2} u^{2}\right)}\right]
\end{aligned}
$$

which is the corresponding characteristic function under the $t$ model, given in Equation (??). 